\title{
Single-Crystal Diamond Nanobeam Waveguide Optomechanics
}

\author{
Behzad Khanaliloo, ${ }^{1,2}$ Harishankar Jayakumar, ${ }^{1, \dagger}$ Aaron C. Hryciw, ${ }^{2,3}$ David P. Lake, ${ }^{1}$ \\ Hamidreza Kaviani, ${ }^{1}$ and Paul E. Barclay, ${ }^{1, *}$ \\ ${ }^{1}$ Institute for Quantum Science and Technology, University of Calgary, \\ Calgary, Alberta, T2N 1N3 Canada \\ ${ }^{2}$ National Institute for Nanotechnology, Edmonton, Alberta, T6G $2 M 9$ Canada \\ ${ }^{3}$ University of Alberta nanoFAB, Edmonton, Alberta, T6G $2 R 3$ Canada
}

(Received 28 April 2015; revised manuscript received 19 October 2015; published 29 December 2015)

\begin{abstract}
Single-crystal diamond optomechanical devices have the potential to enable fundamental studies and technologies coupling mechanical vibrations to both light and electronic quantum systems. Here, we demonstrate a single-crystal diamond optomechanical system and show that it allows excitation of diamond mechanical resonances into self-oscillations with amplitude $>200 \mathrm{~nm}$. The resulting internal stress field is predicted to allow driving of electron spin transitions of diamond nitrogen-vacancy centers. The mechanical resonances have a quality factor $>7 \times 10^{5}$ and can be tuned via nonlinear frequency renormalization, while the optomechanical interface has a $150 \mathrm{~nm}$ bandwidth and $9.5 \mathrm{fm} / \sqrt{\mathrm{Hz}}$ sensitivity. In combination, these features make this system a promising platform for interfacing light, nanomechanics, and electron spins.
\end{abstract}

DOI: 10.1103/PhysRevX.5.041051

Subject Areas: Nanophysics, Photonics

\section{INTRODUCTION}

Nanophotonic optomechanical devices [1-4] enable on-chip optical control of nanomechanical resonators with a precision reaching the standard quantum limit [5-8], enabling tests of quantum nanomechanics [7,9-11], as well as technologies for sensing [4,12-14] and information processing [15-17]. Single-crystal diamond devices are particularly exciting for these applications. In addition to possessing desirable mechanical and optical properties, diamond hosts color centers [18] whose electronic and nuclear spins are promising for quantum technologies.

Recently, nanomechanical devices with embedded color centers [19-23] and quantum dots [24,25] have been used for phononic control [26] of electron spins. These hybrid quantum devices [27] harness the vibrations of nanomechanical resonators to control electron spins via their electronic coupling to internal strain. To date, these experiments have relied on piezoelectric mechanical actuation. Optomechanical devices offer an attractive alternative for mechanical actuation. They use light, the preferred medium for transferring quantum information, to create optical forces that are sensitive to the mechanical resonator position. In combination with coupling between nanomechanical

\footnotetext{
*pbarclay@ucalgary.ca

${ }^{\dagger}$ Present address: Department of Physics, CUNY-City College of New York, New York, New York 10031, USA.

Published by the American Physical Society under the terms of the Creative Commons Attribution 3.0 License. Further distribution of this work must maintain attribution to the author(s) and the published article's title, journal citation, and DOI.
}

resonator strain and electron spins [19-23], this can create an effective interaction between light and spins. Compared to piezoelectric actuation, optomechanical coupling is localized, and offers integrated sensitive readout of mechanical displacement. Although optomechanical devices have been realized from a broad range of materials $[2,6,7,17,28]$, demonstrating single-crystal diamond optomechanical devices has been delayed by challenges in fabrication. Here, we report demonstration of single-crystal diamond optomechanical devices. These devices allow low-power optical actuation of diamond nanomechanical resonators [29-33], and are predicted to enable optical control at wavelengths determined by the device geometry of spin transitions not accessible using traditional radio or microwave frequency techniques [19]. They could also enable proposals for creating hybrid quantum devices for transducing quantum information between disparate quantum systems [34-36], creating quantum sensors [37], generating spin squeezing and entanglement [38], and using quantum emitters for nanomechanical cooling [39,40].

The single-crystal diamond optomechanical system we demonstrate here incorporates several features that together make it a promising platform for hybrid optomechanical quantum devices. This system is based on ultrahigh mechanical quality factor $\left(Q_{m}>7.2 \times 10^{5}\right)$ nanobeam waveguide resonators fabricated from bulk diamond chips using a scalable process. The nanobeams are coupled evanescently to a fiber taper waveguide, and can be excited into self-oscillation via a combination of optomechanical coupling and a stress-enhanced photothermal force that is significant $(>\mathrm{pN})$ even for sub- $\mu \mathrm{W}$ absorbed power levels. We observe self-oscillations with amplitude $>200 \mathrm{~nm}$ for 

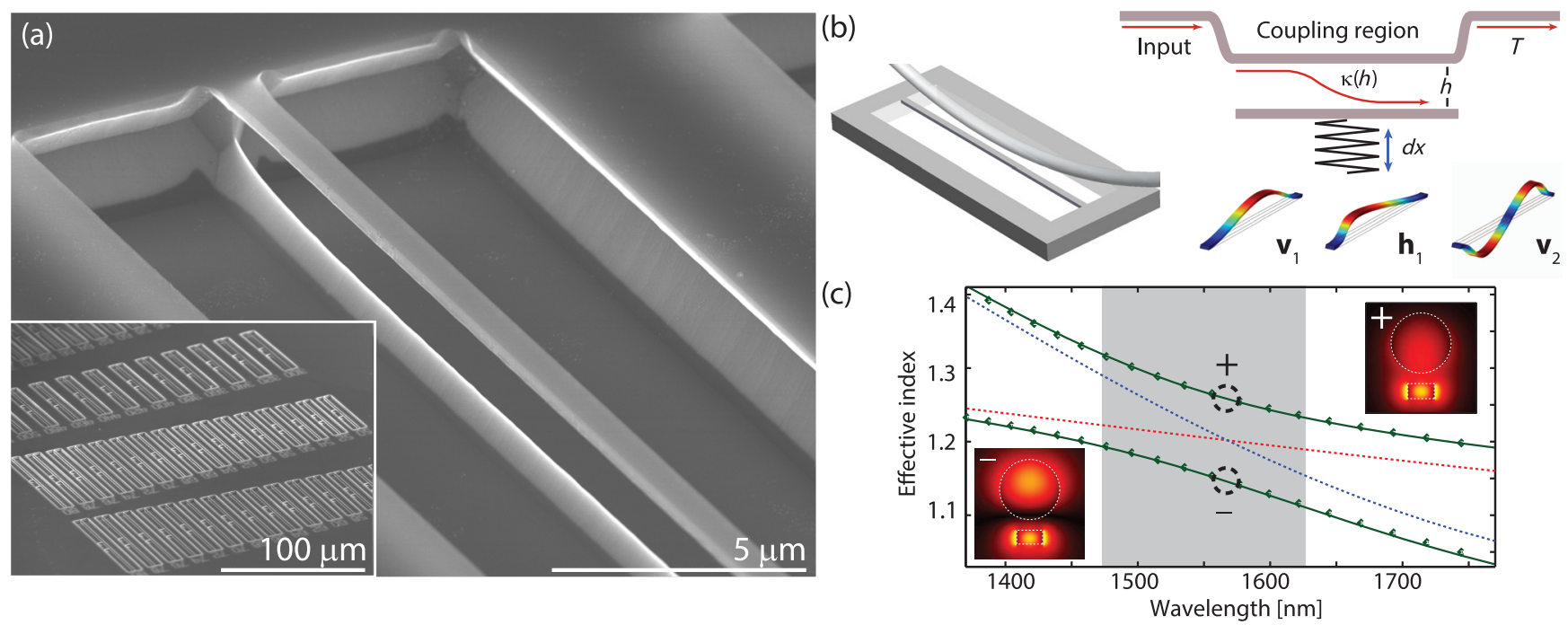

FIG. 1. Diamond nanobeam waveguide-optomechanical system. (a) SEM images of a single-crystal diamond nanobeam waveguide. Dark high-contrast regions are due to variations in thickness of titanium deposited for imaging purposes. (b) Schematic of fiber taper evanescent waveguide coupling geometry and illustration of the waveguide-optomechanical coupling process. Nanobeam mechanical resonances, whose typical displacement profiles are shown, modulate the distance between the input waveguide, changing $\kappa(h)$. (c) Dispersion of $n_{\text {eff }}$ of the waveguide modes of the fiber taper (diameter $\left.1.1 \mu \mathrm{m}\right)$ and diamond nanobeam $\left(w \times d=460 \times 250 \mathrm{~nm}^{2}\right)$ when they are uncoupled (fiber taper shown by red dashed line, nanobeam shown by blue dashed line) and coupled with $h=300 \mathrm{~nm}$ (solid lines). Shaded region indicates the laser scan range used in the experiments. Insets: Mode profiles ( $x-y$ plane, dominant electric field component $\left|E_{y}\right|$ ) of the symmetric (+) and antisymmetric (-) coupled modes.

$\sim 100 \mathrm{nW}$ of absorbed power, and predict that this results in an oscillating internal stress field of $\sim 70 \mathrm{MPa}$. This corresponds to a strain coupling rate of $G / 2 \pi \sim 0.8 \mathrm{MHz}$ to diamond nitrogen-vacancy (NV) center electron spins, indicating that these oscillations can drive coherent transitions between $m_{s}= \pm 1$ states [19-23]. The selfoscillation frequency is observed to be renormalized $200 \mathrm{kHz}$ by nonlinear nanomechanical effects, providing a tunability that would be beneficial for such experiments, as well as a measure of internal stress and nanobeam buckling. The optomechanical interaction underlying this system operates over a $150 \mathrm{~nm}$ bandwidth, and is characterized by a dissipative optomechanical coupling coefficient that can reach $45 \mathrm{GHz} / \mathrm{nm}(0.48 \mathrm{MHz}$ per photon), and a displacement sensitivity of $9.5 \mathrm{fm} / \sqrt{\mathrm{Hz}}$. This sensitivity is less than a factor of 3 above the quantum uncertainty in position of the highest $Q_{m}$ nanobeams demonstrated in this work, and can be improved with further device optimization.

In combination, these properties make the demonstrated system promising for realizing optomechanical spin control, as well as fundamental studies of modification of nanomechanical resonator dynamics by coupling to electronic spins $[39,40]$. The analysis of the device properties and behavior presented here will guide future work to maximize strain coupling to electronic spins, and to harness the large photothermal force for optomechanical cooling and manipulation of nanobeam motion.

\section{WAVEGUIDE-OPTOMECHANICS}

Experiments in diamond nanophotonics and nanomechanics have recently been advanced by the availability of high-quality diamond chips grown using chemical vapor deposition. While optomechanical devices can be fabricated from polycrystalline diamond films [32], single-crystal diamond thin films that retain the desirable combination of optical, mechanical, and quantum electronic properties introduced above are not commercially available and must be manufactured using ion-implantation [41-43] or wafer-bonding $[30,31,44,45]$ techniques. Fabrication of devices directly from bulk single-crystal diamond chips is desirable, and until now has relied on either ion beam milling [46] or less damaging and more scalable inductively coupled plasma reactive-ion (ICPRIE) Faraday cage angled etching $[33,47]$. In this work, we demonstrate an ICPRIE process that does not require a Faraday cage, and instead utilizes diamond undercut etching to fabricate nanobeams from bulk single-crystal diamond, such as those shown in Fig. 1(a) that support optical and mechanical modes summarized in Figs. 1(b) and 1(c), respectively. This process, outlined in Fig. 2 and discussed in more detail in Appendix A, is inspired by earlier bulk silicon nanofabrication [48] and relies on an oxygen-based ICPRIE process operated with zero-rf power, high-ICP power, and an elevated sample temperature $\left(250^{\circ} \mathrm{C}\right)$ to etch diamond quasi-isotropically along diamond crystal planes. The process is uniform over the diamond chip area, can produce 


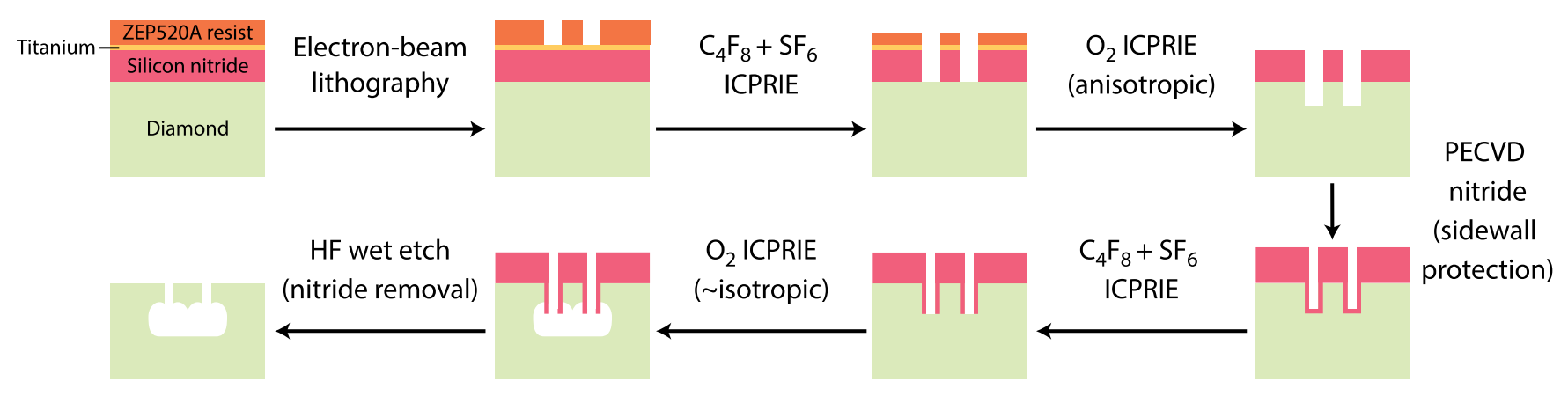

FIG. 2. Process flow for creating diamond nanobeams using quasi-isotropic reactive-ion undercut etching.

horizontal undercut surfaces, and is fully compatible with standard commercial etching tools. Eliminating the need for a Faraday cage allows the process to be employed at a wider range of nanofabrication facilities, while the flat undercutting property allows fabrication of structures such as the high-quality factor microdisk optical cavities in Ref. [49].

Using this diamond undercutting process, nanobeams are fabricated with widths $w=300-540$ and $750 \mathrm{~nm}$ and lengths $L=50-80 \mu \mathrm{m}$ on the same chip. Nanobeam thickness $d$ is adjusted by controlling the etching times together with the nanobeam width $w$. For the etch parameters used here, the nanobeam thickness is $d \sim 250-350 \mathrm{~nm}$, depending on $w$. Because of the crystal-plane sensitive nature of the undercut etch, for the etch parameters used here the narrow nanobeams have a flat bottom surface, while the $w=750 \mathrm{~nm}$ nanobeams have a triangular bottom surface mimicking the ridge visible in Fig. 1(a), as shown in Appendix A. These bottom surfaces can be flattened by longer undercut etching, consistent with observed behavior of microdisks undercut using this process [49].

The fabricated nanobeams support mechanical resonances, the lowest order of which are illustrated in Fig. 1(b), with frequencies and effective mass in the $\mathrm{MHz}$ and pg range, respectively. The nanobeams also support waveguide modes, which can be evanescently coupled with high efficiency to an optical fiber taper [50], as shown schematically in Fig. 1(b), by tuning the device geometry to match the nanobeam mode phase velocity with that of a fiber taper mode. Despite the refractive index difference of the $\mathrm{SiO}_{2}$ fiber taper and diamond, phase-matching is realized in nanobeams with subwavelength cross section. This is illustrated in Fig. 1(c), which shows the effective refractive index dispersion, $n_{\mathrm{eff}}(\lambda)$, of the fundamental TE modes of a fiber taper and of a diamond nanobeam, together with that of the "supermodes" of the evanescently coupled waveguides ( $n_{\text {eff }}^{ \pm}$) [51]. Phase-matching occurs at $\lambda_{0} \sim 1570 \mathrm{~nm}$, where an anticrossing in $n_{\mathrm{eff}}^{ \pm}(\lambda)$ indicates that the waveguide modes are coupled.

Evanescent waveguide coupling, which has been well studied in the context of photonic and optoelectronic devices [51,52], is shown here to provide sensitive optomechanical read-out of mechanical fluctuations that modulate the waveguide separation $h$. In contrast to cavityoptomechanical systems, which exploit dispersive coupling between mechanical fluctuations and narrow-band optical resonances [53], waveguide-optomechanical coupling utilizes wideband dissipative optical transduction of the nanobeam mechanical position. The sensitivity of the evanescent waveguide-optomechanical coupling can be derived from coupled-mode theory (Appendix C) and is determined by the dependence of the normalized fiber taper transmission $T$ on the separation $h$ between the waveguides:

$$
T(h, \lambda)=\cos ^{2}\left(s L_{c}\right)+\left(\frac{\Delta \beta}{2}\right)^{2} \frac{\sin ^{2}\left(s L_{c}\right)}{s^{2}} .
$$

Here, $L_{c}$ is the coupler interaction length determined by the fiber taper geometry, $\Delta \beta(\lambda)=\Delta n_{\text {eff }}(\lambda) 2 \pi / \lambda$ is the propagation constant mismatch of the uncoupled waveguide modes, $s^{2}=\kappa^{2}+\Delta \beta^{2} / 4 . \kappa(h)$ is the per unit length amplitude coupling coefficient, which depends on the overlap of the evanescent fields of the coupled waveguides. The theoretical displacement sensitivity achievable by monitoring fluctuations in $T$ is

$$
s_{x}^{o}(f)=\sqrt{\frac{2 T(h, \lambda) P_{d} \hbar \omega_{o} / \eta_{\mathrm{qe}}+S_{P}^{(\mathrm{det})}}{P_{d}^{2}(\partial T / \partial \kappa)^{2}(\partial \kappa / \partial h)^{2}(\partial h / \partial x)^{2}}},
$$

where $S_{P}^{(\text {det })}$ is the single-sided noise equivalent optical power spectral density (units of $\mathrm{W}^{2} / \mathrm{Hz}$ ) of the detector and technical noise, and $P_{d}$ is the detected output power in the absence of coupling $(T=1)$. The first term in the numerator accounts for photon shot noise, where $\eta_{\mathrm{qe}}$ is the detector quantum efficiency. This expression neglects radiation pressure backaction, which is not significant for the measurements we present here, but will ultimately limit $s_{x}^{o}$. The impact of coupler geometry, operating condition, and waveguide design on detection sensitivity is described by the denominator of Eq. (2). Sensitivity is maximized for mechanical resonances whose displacement $x$ efficiently modulates $h$, i.e., $|\partial h / \partial x| \sim 1$. Strong evanescent overlap 
enhances $|\partial \kappa / \partial h|$, while phase-matching and operation near $\kappa(h) L_{c} \sim \pi / 4,3 \pi / 4, \ldots$ maximizes $|\partial T / \partial \kappa|$.

Experimental observation of the optomechanical properties of the diamond waveguide-optomechanical system is performed by measuring $T(t)=\bar{T}+\delta T(t)$ of a dimpled optical fiber taper [50] positioned in the nanobeam nearfield for varying $h$ and $\lambda$. The efficiency of the evanescent coupling determines the time-averaged transmission $\bar{T}$. Fluctuations of the nanobeam position, together with other noise, are imprinted on $\delta T(t)$. Figure 3(a) shows $\bar{T}(\lambda)$ when the fiber taper is positioned at $h \sim 200 \mathrm{~nm}$ above the center of a nanobeam $\left(w \times d=460 \times 250 \mathrm{~nm}^{2}\right)$. Minimum transmission $\bar{T}_{o}=0.05$, corresponding to a coupling efficiency of $1-\bar{T}_{o}=0.95$ assuming negligible insertion loss, is observed near $\lambda_{0}=1560 \mathrm{~nm}$. The $3 \mathrm{~dB}$ bandwidth $\Delta \lambda>$ $150 \mathrm{~nm}$ is consistent with predictions from the $n_{\mathrm{eff}}^{ \pm}(\lambda)$ anticrossing in Fig. 1(c), and increasing $w$ is observed to increase $\lambda_{0}$ [Fig. 3(a), inset], consistent with expected behavior.

The coherent nature of the waveguide coupling is revealed by $\bar{T}_{o}(h)$. As shown in Fig. $3(\mathrm{~b}), \bar{T}_{o}(h)$ is minimized at $h \sim 200 \mathrm{~nm}$, where $\kappa L_{c}=\pi / 2$. For $h<200 \mathrm{~nm}, \bar{T}_{o}$ increases with decreasing $h$ due to the codirectional coupling undergoing more than a half "flop" and light coupled into the nanobeam being outcoupled back
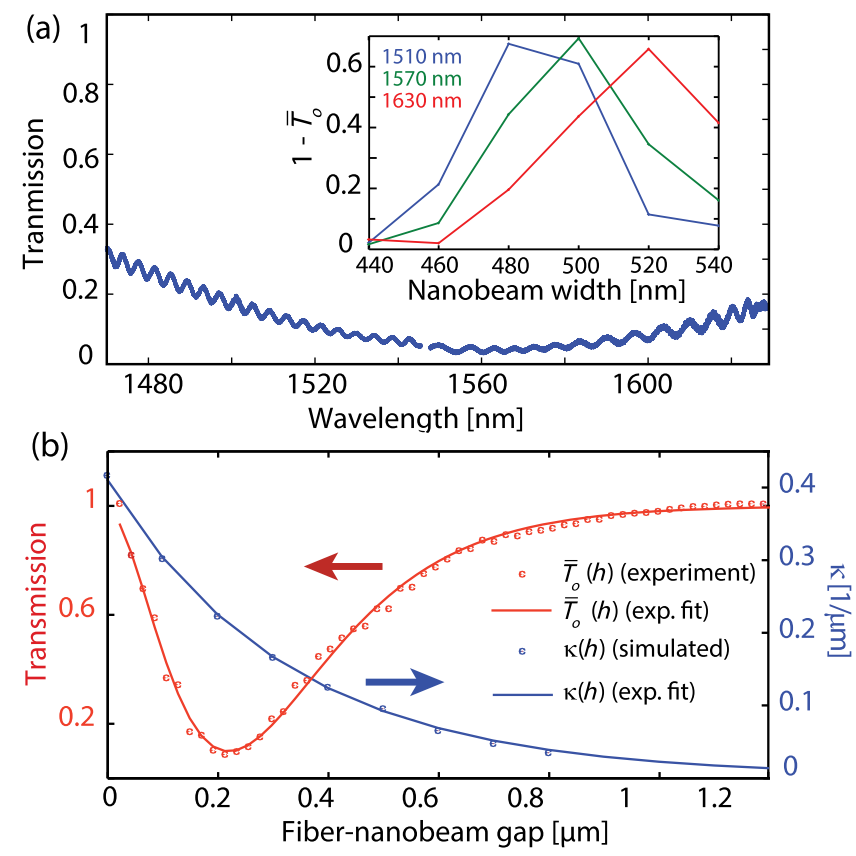

FIG. 3. Efficient evanescent waveguide coupling. (a) Fiber taper transmission $\bar{T}(\lambda)$ versus wavelength for $h=200 \mathrm{~nm}$. Inset: Variation of $\bar{T}$ with nanobeam width $w$ and $\lambda$ for approximately constant $h$. (b) Dependence of fiber taper transmission on fiber height. Experimentally measured values for $\bar{T}_{o}$ (red points) are fit with the model described by Eq. (1) (red line). The corresponding $\kappa(h)$ extracted from the fit (blue line) and predicted from simulation (blue points) are also shown. into the fiber taper [52]. In contrast, incoherent scattering loss increases monotonically with decreasing $h$ [54] and is small in the system studied here. As shown in Fig. 3(b), Eq. (1) fits $\bar{T}_{o}(h)$ well with an exponentially decaying $\kappa(h)$ as a fitting parameter, which in turn agrees closely with $\kappa(h)$ predicted from coupled-mode theory for interaction length $L_{c} \sim 7 \mu \mathrm{m}$. This $L_{c}$ is consistent with the observed curvature in optical images of the fiber taper dimple.

The optomechanical properties of the coupled waveguides are observed from the power spectral density $S_{v}(f)$ of the photodetected signal generated by fluctuations in output power $P_{d} \delta T(t)$. Figure 4(a) shows the equivalent displacement spectral density $s_{x}(f)$ (units $\mathrm{m} / \sqrt{\mathrm{Hz}}$ ) of the nanobeam motion when the fiber taper is positioned $h \sim 100 \mathrm{~nm}$ above the nanobeam. Peaks from thermally driven nanobeam resonances are clearly visible at frequencies $f_{m}=[1.3,3.1,4.2] \mathrm{MHz}$, corresponding to the fundamental out-of-plane $\left(\mathbf{v}_{1}\right)$, fundamental in-plane $\left(\mathbf{h}_{1}\right)$, and second-order out-of-plane $\left(\mathbf{v}_{2}\right)$ resonances, whose simulated displacement profiles are shown in Fig. 1(b). Resonance labels are determined by comparison of $f_{m}$ with simulations and by measuring transduction sensitivity as a function of in-plane taper position. Assuming a $35 \mathrm{MPa}$ internal compressive stress, consistent with studies of the nanobeam response described in the following section, we find simulated values of $1.3,3.2$, and $4.2 \mathrm{MHz}$, in good agreement with observed values. $s_{x}(f)$ is obtained by calibrating $S_{v}(f)$ to the theoretical thermomechanical power spectral density of the $\mathbf{v}_{1}$ resonance $(m=7.6 \mathrm{pg}$ ), as described in Appendix E. The observed technical noise floor of $s_{x}^{o}=9.5 \pm 1.0 \mathrm{fm} / \mathrm{Hz}^{1 / 2}$ is below that of other broadband integrated waveguide measurements $[32,54,55]$, and is more than an order of magnitude more sensitive than single-pass free-space reflection measurement techniques used with diamond nanomechanical structures [33]. Fiber interferometers incorporating low-finesse optical cavities $[56,57]$ have reached similar sensitivity. This sensitivity can be further improved to the sub-fm/ $/ \mathrm{Hz}^{1 / 2}$ range by increasing $L_{c}$ and $P_{d}$, or by terminating the nanobeams with photonic crystal mirrors to introduce optical feedback at the expense of optical bandwidth, as in the multipass cavityfiber-interferometer system of Ref. [58]. Uncertainty in $s_{x}^{o}$ is derived from uncertainty in $m$ resulting from possible variations from the nominal device geometry and material parameters.

The $h$ dependence of $S_{v}\left(f_{m}\right)^{1 / 2}$ and $s_{x}^{o}$ for the $\mathbf{v}_{1}$ resonance, shown in Fig. 4(b), provides a direct measure of the optomechanical coupling. The on-resonance signal is directly related to the slope of $\bar{T}(h): S_{v}\left(h ; f_{m}\right)^{1 / 2} \propto$ $|d \bar{T} / d h|$, with a distinct minimum when $\kappa L_{c}=\pi / 2$. Highest sensitivity is observed at $h=100 \mathrm{~nm}\left(\kappa L_{c}=\right.$ $3 \pi / 4)$, and $s_{x}^{o}(h)$ agrees well with predictions from the experimentally characterized $\bar{T}(h)$ and Eq. (2). As described in Appendix E, the predicted sensitivity assumes a shot-noise-limited laser source over the measurement 

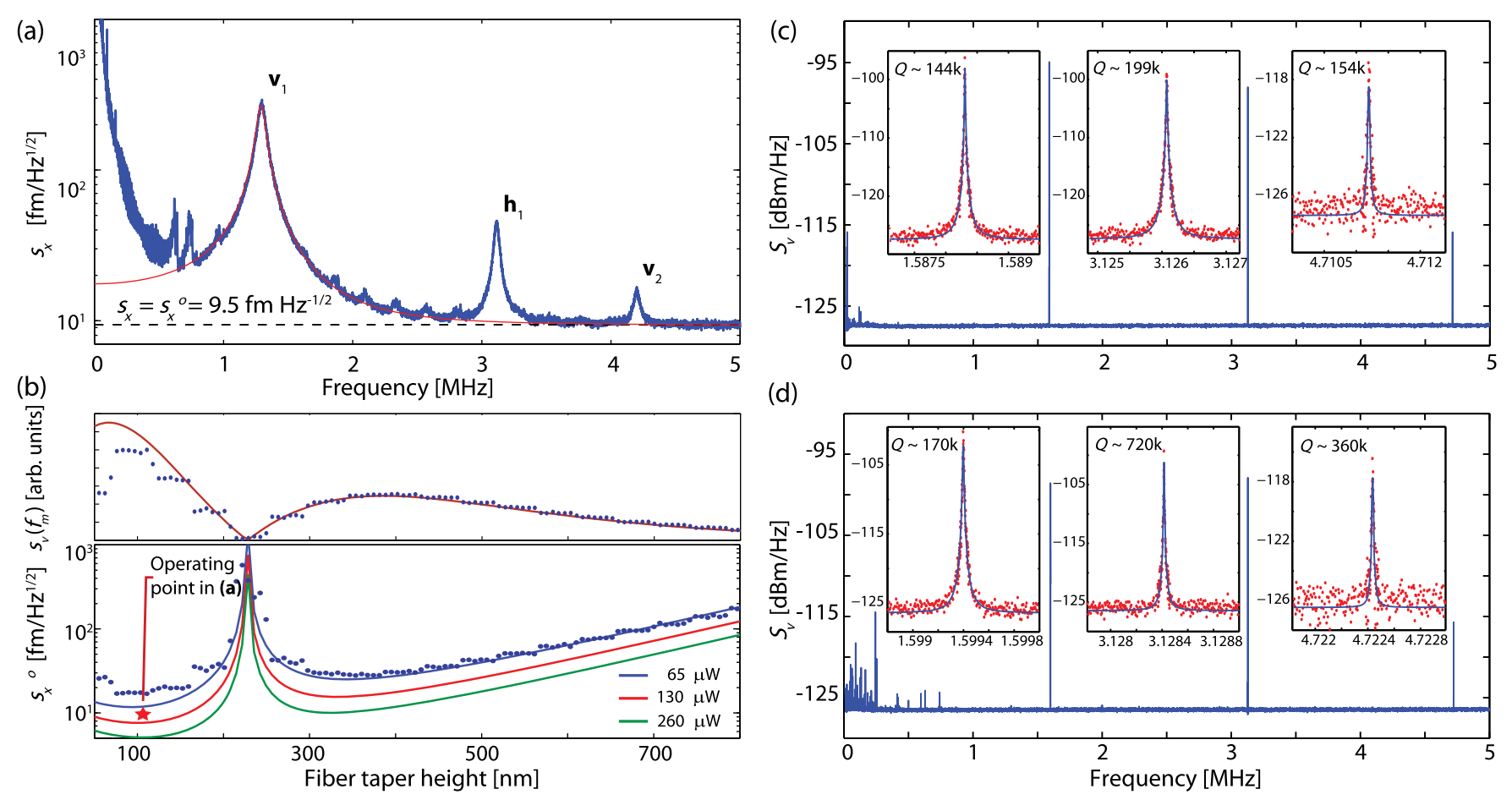

FIG. 4. Nanomechanical and optomechanical properties. (a) Measured $s_{x}(f)$ when the fiber taper is coupled to a nanobeam $\left(L \times w \times d=50 \times 0.46 \times 0.25 \mu \mathrm{m}^{3}\right)$ in ambient conditions, with $P_{d} \sim 100 \mu \mathrm{W}$. The vertical axis and noise floor (dashed line) of $s_{x}^{o}=9.5 \mathrm{fm} / \sqrt{\mathrm{Hz}}$ are calibrated from the fit to the $f=1.3 \mathrm{MHz}$ mechanical resonance thermomechanical displacement spectrum (red solid line). (b) Top: $S_{v}\left(f_{1}\right)^{1 / 2}$ observed (points) and fit with a function $\propto|d \bar{T} / d h|$ (solid line). Bottom: Displacement sensitivity $s_{x}^{o}(h)$ of the fundamental out-of-plane resonance from measurement (blue points) and predicted from the measured $T(h)$ for varying input power (solid lines). The starred point corresponds to the sensitivity and operating condition of the measurement in (a). Measured $S_{v}(f)$ for a nanobeam with $L \times w \times d=60 \times 0.75 \times 0.3 \mu \mathrm{m}^{3}$ (c) in vacuum (d) at $5 \mathrm{~K}$. Insets: Fits to observed mechanical resonances, and corresponding $Q_{m}$. Unlabeled peaks in (a), (c), and (d) below $1 \mathrm{MHz}$ are related to fiber vibrations.

bandwidth of interest (i.e., the $\mathrm{MHz}$ range) and a photodetector noise equivalent power $S_{P}^{(\mathrm{det})}=2.5 \mathrm{pW} / \sqrt{\mathrm{Hz}}$ corresponding to the nominal specification for the detector used here. The minimum $s_{x}^{o}(h)$ in Fig. 4(b) is degraded compared to the sensitivity of the measurement in Fig. 4(a) due to operating at lower $P_{d}$.

As discussed in Appendix D, the optomechanical transduction $d \bar{T} / d h$ is related to the dissipative optomechanical coupling coefficient $g_{\gamma}^{(e)}$, defined by the optomechanical modulation of tunneling rate $\gamma_{e}$ of photons from the nanobeam to the forward propagating fiber taper mode. Assuming the coupler demonstrated here is integrated into a cavity of length $L=L_{c}$, we predict $g_{\gamma}^{(e)} / 2 \pi>$ $45 \mathrm{GHz} / \mathrm{nm}$, where $c / n_{g}$ is the group velocity of light in the coupler. The corresponding per photon optomechanical coupling rate is $g_{o} / 2 \pi \sim 0.48 \mathrm{MHz}$ for the $\mathbf{v}_{1}$ resonance of a nanobeam of length $L_{c}$ and cross section of the device in Fig. 4(b) $\left(f_{m} \sim 66 \mathrm{MHz}\right.$, zero-point motion $\left.x_{\mathrm{zpf}}=11 \mathrm{fm}\right)$. In addition to being tunable via adjustment of $h$, this dissipative coupling is significantly larger than in previously studied systems [59-62]. However, note that when the dissipative coupling is maximized, it is accompanied by large $\gamma_{e}$. Also note that the coupler introduces dispersive coupling, discussed in Appendix D, which is small compared to $g_{\gamma}^{(e)}(h)$ for $h>200 \mathrm{~nm}$.

Unlike dispersive optomechanical coupling, dissipative optomechanics [59] can be used to cool nanomechanical resonances in cavity-optomechanical systems operating in the sideband-unresolved regime $[63,64]$. This is a potentially useful tool for optomechanical cooling of relatively low-frequency nanomechanical resonances. Realizing dissipative cooling of the devices studied here would require further device fabrication development in order to integrate the fiber-coupled nanobeam into a cavity. Furthermore, as discussed in Appendix D, it is necessary that the cavity mode can be coupled unidirectionally [53] to the fiber taper waveguide via the nanobeam [64] and have internal loss rate $\gamma_{i} \ll \gamma_{e}$. Oval racetrack resonators similar to those demonstrated by Burek et al. [47], modified to incorporate clamped diamond nanobeams with $L=L_{c}=21 \mu \mathrm{m}$ a supporting traveling-wave resonance, are promising in this regard. We predict in Appendix D that over a narrow range of $h$, which balances small $\gamma_{e}$ and large $g_{\gamma}^{(e)}$, optomechanical reduction of the fundamental nanobeam normal-mode phonon population to close to $10^{2}$ quanta, limited by optomechanical backaction, may be possible in such a 
device if an internal loss rate of $\gamma_{i} / 2 \pi=1 \mathrm{GHz}$ and an internal backscattering rate less than $\gamma_{i}$ can be achieved.

Nanobeam mechanical dissipation in the measurements discussed above is dominated by damping from the ambient air environment. To assess the nanobeam mechanical properties, measurements are performed in vacuum and low-temperature conditions. Generally, after reducing air damping, $Q_{m}$ is observed to increase above $10^{4}$ in all device geometries, with the lowest dissipation $\left(Q_{m}>10^{5}\right)$ observed in the larger cross-section $(w \times d=750 \times$ $300 \mathrm{~nm}^{2}$ ) devices. Figures 4(c) and 4(d) show mechanical resonances of a high- $Q_{m}$ nanobeam measured in vacuum (80 $\mu$ Torr) and in cryogenic conditions $(T=5 \mathrm{~K}$ and $5 \mu$ Torr). In vacuum, the $\mathbf{v}_{1}$ and $\mathbf{h}_{1}$ resonances have $Q_{m} \approx$ $1.4 \times 10^{5}$ and $2.0 \times 10^{5}$, respectively. At low temperatures, dissipation is further reduced, such that $Q_{m} \approx 1.7 \times 10^{5}$ and $7.2 \times 10^{5}$ for the $\mathbf{v}_{1}$ and $\mathbf{h}_{1}$ modes, respectively. These values of $Q_{m}$ are higher than previous reports of nanomechanical devices fabricated from single-crystal optical grade diamond $[30,31,33]$. All measurements are made at low power to avoid inducing optomechanical linewidth narrowing, and spectral diffusion is not observed on the few-second measurement time scale. The observed increase in $Q_{m}$ with increasing nanobeam mode order and type ( $\mathbf{v}$ versus $\mathbf{h})$, together with data presented below for a larger $L$, smaller cross-section nanobeam with lower $Q_{m}$ than the device in Figs. 4(c) and 4(d), indicates that clamping loss is not the dominant dissipation mechanism for these structures [65]. This behavior, and other possible sources of loss including surface dissipation [31], requires further investigation in order to understand how to increase $Q_{m}$ in future devices. Two possible approaches include fabricating devices from electronic grade material or with larger dimensions [31].

Given the demonstrated device performance, the measurement precision required to reach the standard quantum limit is $s_{x}^{\mathrm{SQL}}=\sqrt{\hbar Q_{m} /\left(m \omega_{m}^{2}\right)}=3.1 \mathrm{fm} / \sqrt{\mathrm{Hz}}$, where $m=23 \mathrm{pg}$ is the effective mass of the $\mathbf{h}_{1}$ resonance [9]. While this is $\sim 3$ times smaller than the measurement sensitivity demonstrated here, promising approaches for reducing $s_{x}^{o}$ below $s_{x}^{\mathrm{SQL}}$ include improving the optomechanical coupling by increasing $L_{c}$, and fabricating higher$Q_{m}$ devices.

\section{TUNABLE NONLINEAR DYNAMICS}

Backaction from waveguide-optomechanical coupling can dramatically modify the nanobeam dynamics, and is shown here to amplify the nanobeam motion and reveal nonlinear nanomechanical properties of the device. These effects can be tuned by adjusting the waveguide position, and depend critically on the presence of internal stress in the nanobeam. Demonstration of optomechanically modified nanobeam dynamics is shown in Fig. 5(a), where time-resolved $T(t)$ of a fiber taper coupled to a high-aspectratio nanobeam $\left(L \times w \times d=80 \times 0.48 \times 0.25 \mu \mathrm{m}^{3}\right)$ in a vacuum environment is recorded while $h$ is discretely stepped $\left(30 \mathrm{~nm} / \mathrm{step}, 0.8\right.$ steps $\left./ \mathrm{s}, P_{i} \sim 300 \mu \mathrm{W}\right)$. For large $h, T_{o}(h)$ behaves similarly to the ambient condition measurements in Fig. 4(a). However, at $h \sim 400 \mathrm{~nm}$ fluctuations $\delta T(t)$ become large-amplitude self-oscillations with a peak-to-peak change $\Delta T>0.3$, corresponding to nanobeam displacement exceeding $200 \mathrm{~nm}$, as illustrated in the inset of Fig. 5(a). To the best of our knowledge, this amplitude is larger than in previous reports of on-chip externally driven single-crystal [66] and polycrystalline [32] diamond nanobeams. Note that calibration of the observed nanobeam mechanical displacement is enabled by the tunable nature of the optical coupling and the use of encoded nanopositioning of the fiber taper. Finite element simulations predict a variation of axial stress at the center of the nanobeam on the order of $70 \mathrm{MPa}$ for the $\mathbf{v}_{1}$ resonance of the compressed nanobeam oscillating with this amplitude, corresponding to a strain to $\mathrm{NV}$ electronic spin-coupling rate of $G / 2 \pi \sim 0.8 \mathrm{MHz}$, enabling optomechanical control of their quantum state [20,21]. When $h<225 \mathrm{~nm}$, the self-oscillations stop. Figure 5(b) shows a spectrograph of these data, where the $\mathbf{v}_{1}$ resonance near $f_{m}=430 \mathrm{kHz}$ with $Q_{m}=2.5 \times 10^{4}$ is observed to increase in amplitude, shift to lower frequency, and generate harmonics.

Unlike previously observed diamond nanobeam oscillations driven by electrostatic [66] or optical modulation [32], the observed oscillations are regenerative in nature. These self-oscillations are driven by the dynamic interaction between a delayed photothermal force $[67,68]$ and the waveguide-optomechanical coupling. They have not been previously observed in waveguides, and can possess large amplitudes owing to the large dynamic range and bandwidth of the waveguide-optomechanical system. The resulting optomechanical backaction renormalizes the mechanical dissipation rate from $\gamma_{m}$ to $\gamma_{m}^{\prime}$,

$$
\frac{\gamma_{m}^{\prime}}{\gamma_{m}}=1-Q_{m} \frac{\mathcal{F}}{k} \frac{\omega_{m} \tau}{1+\omega_{m}^{2} \tau^{2}} \frac{d T}{d x} P_{i} \zeta L_{i},
$$

where $\tau$ is the photothermal force response time and $\mathcal{F}$ is the photothermal force strength per unit absorbed power $P_{\text {abs }}=(1-T) P_{i} \zeta L_{i}$, defined by the corresponding nanobeam deflection and spring constant $k=m \omega_{m}^{2}$ of the mode of interest. $L_{i}$ is the distance over which input light propagates in the nanobeam before being outcoupled and $\zeta$ is the waveguide absorption coefficient per unit length. Figure 5(c) compares experimentally observed $\gamma_{m}^{\prime}(h)$ with theoretical values obtained from Eq. (3) input with measured $T(h)$ as well as parameters discussed below. Predicted $\gamma_{m}^{\prime}(h)$ agrees well with experiment, particularly in reproducing the range of $h$ over which the optomechanical coupling $(|d T / d x|)$ is sufficiently large such that $\gamma_{m}^{\prime}<0$, resulting in self-oscillations. 

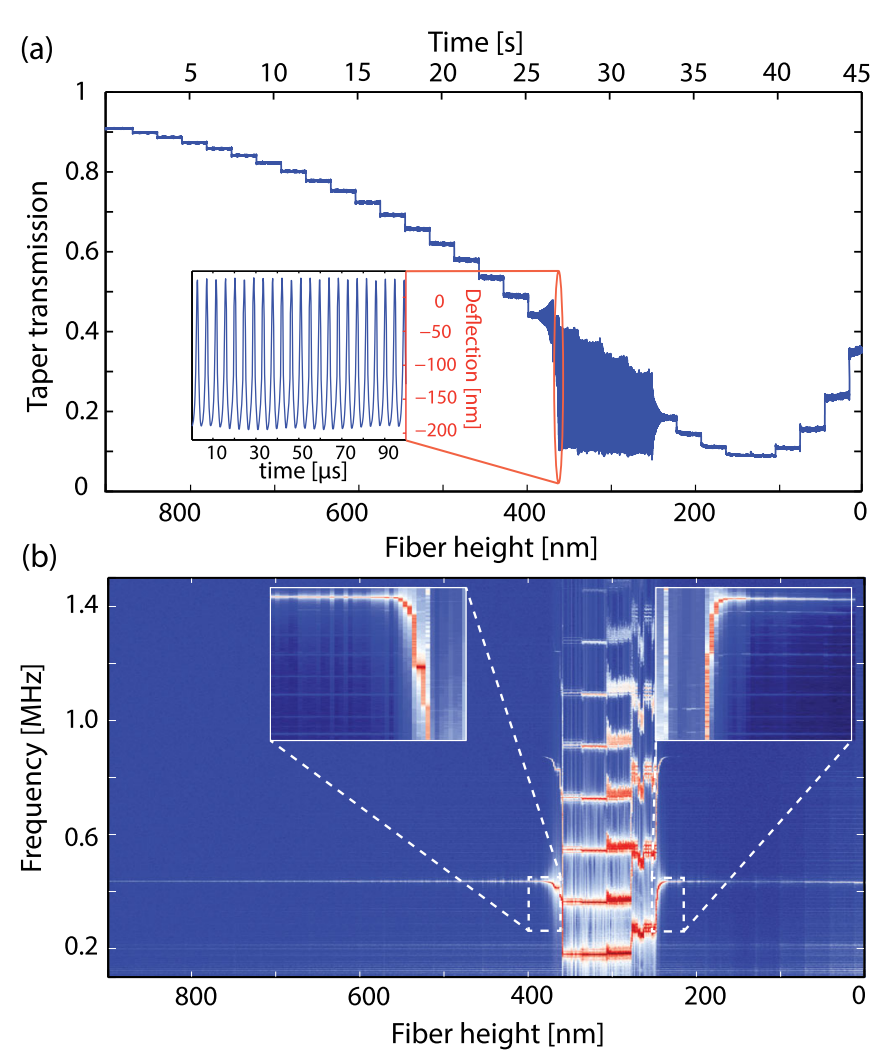

(c)
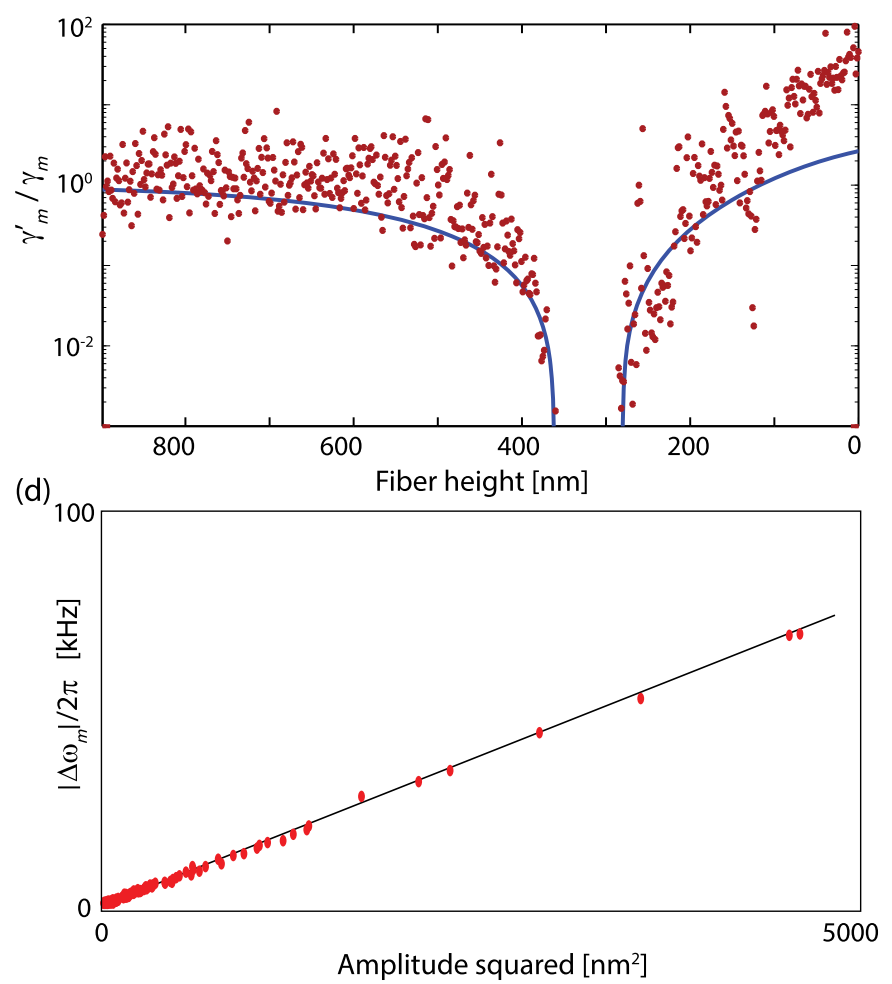

FIG. 5. Nanobeam self-oscillations. (a) Time-resolved $T(h(t))$ of a phase-matched fiber taper nanobeam $(L \times w \times d=$ $80 \times 0.50 \times 0.25 \mu \mathrm{m}^{3}$ ) system as $h$ is reduced in discrete steps (visible as sharp steps in $T$ ). Inset: Large-amplitude oscillation of the nanobeam position. (b) Spectrograph showing the power spectral density of the data in (a). (c) Predicted renormalized mechanical dissipation rate compared with measured values. (d) Scatter plot of shift in nanobeam resonance frequency versus oscillation amplitude squared for data in the inset regions from (b) at the onset of self-oscillation. The solid line is a linear guide to the eye.

For $h<200 \mathrm{~nm}$, photothermal damping broadens $\gamma_{m}$. However, coupling between $\omega_{m}$ and the fiber taper position for this range of $h$ (see Appendix G), combined with fluctuations in fiber position due to external vibrations, artificially broadens $\gamma_{m}$ and makes accurate measurement of the cooling rate challenging. If $P_{i}$ were increased to $3 \mathrm{~mW}, \gamma_{m}^{\prime} / \gamma_{m} \sim 10$ is theoretically possible. If applied to a higher- $Q_{m}$ device, such as the structures in Fig. 4(d), larger photothermal normal-mode cooling may be possible at this power level.

In addition to exhibiting high $Q_{m}$ and strong dissipative optomechanical coupling, the nanobeams have two properties that make them sensitive to optomechanical photothermal actuation. The nanobeam thermal time constant $\tau \sim 0.7 \mu \mathrm{s}$, calculated using finite element simulations, is on the same time scale as $\omega_{m}$. As seen from Eq. (3), this is a necessary condition for significant feedback from the photothermal force [67-70]. A more subtle but equally important property is the presence of compressive stress and accompanying buckling in the nanobeam, which as discussed below, dramatically enhances $\mathcal{F}$.

For the nanobeam geometry studied here, finite element analysis presented in Appendix $\mathrm{G}$ indicates that $\mathcal{F}$ can be enhanced by over 2 orders of magnitude in a compressed and buckled nanobeam. The level of compressive stress in a nanobeam can be estimated from the deviation of $\omega_{m}$ from the nominal value expected for an uncompressed device [33]. The nanobeam we study in Fig. 5 is observed to be in a postbuckled state with measured $\omega_{m} / 2 \pi \sim 430 \mathrm{~Hz}$, lower than the nominal value expected $(680 \mathrm{kHz})$ for an uncompressed nanobeam. Matching finite element simulated and observed $\omega_{m}$ predicts an internal compressive stress of $\sim 37 \mathrm{MPa}$ and an accompanying buckling amplitude of $\bar{x}=-122 \mathrm{~nm}$. For this value of internal stress and buckling, a photothermal force of $\mathcal{F}=-26 \mathrm{pN} / \mu \mathrm{W}$ is predicted, which is over 100 times larger than predicted for an uncompressed device, and as a result is significant even for low optical absorption within the nanobeam. This enhancement originates from more efficient conversion of axial stress to transverse deflection in buckled nanobeams (see Eq. (G7)), and is also present to a lesser degree in compressed prebuckled nanobeams with small amounts of bending. This effect may be effective for photothermal actuation of compressed optomechanical crystal [2] and ring or racetrack devices [47]. The negative signs of $\mathcal{F}$ and $\bar{x}$ indicate that the photothermal force and buckling amplitude are downward, consistent with all of the selfoscillation behavior discussed in this section. Note that the 
buckled nanobeam shape and resulting variation of $h$ along the nanobeam length is not included in waveguide coupling calculations since this curvature is small compared with that of the dimpled fiber taper.

Given the above device parameters (summarized in Table I of Appendix G), the only free-fitting parameter needed to match Eq. (3) with experiment is the waveguide absorption coefficient, which is found to be $\zeta=0.12 \mathrm{~cm}^{-1}$, corresponding to an optical loss rate effective quality factor of $Q_{o} \sim 6.6 \times 10^{5}$. This absorption rate is consistent with loss in other diamond nanophotonic devices fabricated with both this process [49] and other techniques [47], and indicates that only a fraction $(\sim 100 \mathrm{nW})$ of the input power is absorbed and responsible for driving the self-oscillations. This absorbed power level corresponds to an average of approximately 0.04 photons absorbed per transit time of the coupler region. While small, this absorption rate is higher than expected in bulk diamond. This is possibly a result of etching-related surface state absorption or impurities in the diamond, and requires further investigation.

Nonlinear coupling between nanobeam oscillation amplitude and $\omega_{m}$ provides an additional probe of the internal stress and buckled state of the device. The high sensitivity and the time-resolved absolute measurement of nanobeam position provided by the waveguide-optomechanical system allows nanobeam resonance frequency to be studied as a function of oscillation amplitude. Nonlinear softening of the mechanical resonance frequency by $\Delta \omega_{m}(h)$ is observed at the onset of self-oscillations, as highlighted in Fig. 5(b). Nanobeam softening and hardening is an indicator of internal stress $[32,66]$, and the softening observed here is found to be closely related to the buckled nanobeam state. Nanobeam buckling breaks the device vertical symmetry and introduces a nonlinear softening term to the nanobeam dynamics, which counteracts the hardening effect described by the intrinsic Duffing nonlinearity [71]. This competition between nonlinear effects is given by

$$
\Delta \omega_{m}=\frac{v^{2}}{\omega_{m}}\left(\frac{3}{8} \alpha_{3}-\bar{x}^{2} \frac{15}{4 \omega_{m}^{2}} \alpha_{3}^{2}\right),
$$

where $v$ is the oscillation amplitude and $\alpha_{3}$ is the Duffing coefficient of the unbuckled nanobeam, as described in Appendix G. Equation (4) clearly shows the softening influence of $\bar{x}$. The optomechanical system we study here provides a direct measurement of $\Delta \omega_{m}$ and $v$ for varying $h$, allowing $\bar{x}$ to be estimated experimentally. Figure 5(d) shows a scatter plot of measured $\Delta \omega_{m}$ as a function of $v^{2}$ for varying fiber taper position. As predicted from Eq. (4), $\Delta \omega_{m}$ and $v^{2}$ are found to be linearly related, and from these data and Eq. (4), $\bar{x}=-98 \mathrm{~nm}$ is estimated, which is in excellent agreement with finite element simulation predictions of $\bar{x}$ given above. This approach for simultaneously actuating nanomechanical motion and directly monitoring the nonlinear response of nanomechanical devices can easily be applied to other material systems. For example, fiber taper coupling to silicon and other semiconductor nanowire [72] and photonic crystal [52] waveguides is possible.

During the self-oscillation limit cycle, a low fundamental self-oscillation frequency of $\sim 180 \mathrm{kHz}$ is measured, consistent with the behavior of a nanomechanical resonator oscillating between buckled states, as observed by Bagheri et al. [15]. Further evidence of this behavior is found in Fig. 5(a), which shows that during self-oscillations $\bar{T}$ decreases, indicating that the nanobeam, initially in a buckled down state, moves on average closer to the fiber taper while self-oscillating. Note that harmonics which emerge during self-oscillation, becoming stronger and more numerous as $v$ increases, result from both the nonlinear response of $\bar{T}(h)$ and nonlinearities of the nanobeam [73].

\section{DISCUSSION AND CONCLUSION}

We have demonstrated an optomechanical system from single-crystal diamond and have shown that it possesses a unique combination of high sensitivity, broad bandwidth, high-quality single-crystal diamond material, high $Q_{m}$, and low-power self-oscillation threshold. It has potential to enable measurement of quantum motion of nanobeam resonances and fundamental studies and technologies based on hybrid quantum devices. Excitation of nanomechanical self-oscillations with $\mathrm{nW}$ absorbed power illustrates the sensitivity of the diamond nanobeams to small driving forces, and their nonlinear dynamical softening provides a glimpse of the changing stress within the nanobeam during large-amplitude oscillations. This demonstration of optomechanical excitation of the diamond nanomechanical environment is a step towards control of quantum electronic systems such as nitrogen vacancies using optomechanical actuation of classical nanomechanical oscillators [19-21,26,38]. Future devices may be designed to operate at visible $(637 \mathrm{~nm})$ wavelengths resonant with NV center optical transitions, creating additional opportunities for realizing hybrid quantum devices.

The detailed analysis and understanding of the devices presented here allows prediction of the internal strain dynamics and self-oscillation threshold of nanobeams used for creating hybrid quantum systems. This analysis reveals that the threshold can be surprisingly low, and that photothermal forces do not require large absorption or an optical cavity to introduce significant backaction in the waveguideoptomechanical system. This understanding will guide further optimization of device geometry and operating conditions needed to improve the measurement sensitivity in order to resolve quantum motion of the nanobeam and to harness the photothermal forces for optomechanical cooling. Reducing the nanobeam length may allow coupling to nanomehanical modes with frequencies sideband resolved from spin transitions or resonant with higher-energy diamond nitrogen-vacancy spin transitions tuned by external 
magnetic fields $[19,34,38]$. If the nanobeams studied in Figs. 4(c) and 4(d) were shortened to $L=L_{c}=7 \mu \mathrm{m}$, the three lowest-order resonances would shift to $f_{m} \sim 135,275$, and $366 \mathrm{MHz}$, and the absolute optomechanical read-out sensitivity would remain constant. However, note that the photothermal actuation would become less efficient [see Eq. (G7)], and resonant optical modulation may be required to drive large-amplitude oscillations of these nanobeams.

This system is also promising for implementations of optically actuated classical logic based on buckled nanomechanical states [15] and can be extended to use the optical gradient force for optomechanical nanobeam actuation, enabling excitation of higher-frequency resonances of smaller structures [2]. Finally, the scalable nanofabrication technique demonstrated here is widely applicable to diamond nanophotonic devices for sensing, nonlinear optics, and quantum information processing, and can be easily adopted by researchers with access to standard nanofabrication tools. Extending this approach to integrate the waveguide-optomechanical system into an optical cavity, for example, a racetrack resonator [47], may allow dissipative optomechanical cooling of the nanobeam resonances [64]. Similarly, replacing the fiber taper with an on-chip waveguide optomechanically coupled to the nanobeam may be possible; such a system will retain the features demonstrated here, trading stability inherent to monolithic systems in exchange for tunability provide by fiber taper coupling.

\section{ACKNOWLEDGEMENTS}

We thank Charles Santori and David Fattal for useful initial discussions related to the fabrication approach used here. We would like to acknowledge support for this work from NSERC, iCore/AITF, CFI, NRC.

\section{APPENDIX A: FABRICATION PROCESS}

The fabrication process flow is outlined in Fig. 2. A chemical-vapor-deposition-grown, $\langle 100\rangle$-oriented singlecrystal diamond optical grade substrate (Element Six) is cleaned in boiling piranha $\left(3: 1 \mathrm{H}_{2} \mathrm{SO}_{4}: \mathrm{H}_{2} \mathrm{O}_{2}\right)$ and coated with a $300 \mathrm{~nm}$ thick layer of plasma-enhanced chemical vapor deposition (PECVD) $\mathrm{Si}_{3} \mathrm{~N}_{4}$ as a hard mask. Next, 2-5 nm of titanium is deposited as an anticharging layer. Nanobeam structures with axes aligned along the $\langle 110\rangle$ direction are patterned in ZEP520A electron-beam lithography resist and developed at $-15^{\circ} \mathrm{C}$ in ZED-N50. The resulting ZEON electron-beam positive (ZEP) resist pattern is transferred to the nitride hard mask using an inductivelycoupled plasma reactive-ion etch (ICPRIE) process with $\mathrm{C}_{4} \mathrm{~F}_{8} / \mathrm{SF}_{6}$ chemistry. An anisotropic oxygen plasma ICPRIE step transfers the pattern to the diamond, followed by a $160 \mathrm{~nm}$ thick conformal coating of PECVD $\mathrm{Si}_{3} \mathrm{~N}_{4}$ to protect the vertical diamond sidewalls. A short anisotropic $\mathrm{C}_{4} \mathrm{~F}_{8} / \mathrm{SF}_{6}$ ICPRIE step clears the nitride from the bottom of the windows while keeping the side and top surface of the devices protected. To create suspended nanobeams, a quasiisotropic oxygen plasma etch [74] is performed at $2500 \mathrm{~W}$ ICP power, $0 \mathrm{~W}$ rf power, and elevated wafer temperature of $250^{\circ} \mathrm{C}$. This etch step is relatively slow, requiring $\sim 5 \mathrm{~h}$ to undercut the nanobeams studied here. Figure 6(a) shows cross sections of fabricated devices with varying nominal widths. The undercut etch rate is observed to increase at higher ICP power; however, this option is not available for the devices fabricated for this report. An Oxford Plasmalab is used for all plasma etch steps. Finally, the titanium and nitride layers are removed by wet-etching in $49 \% \mathrm{HF}$, and the sample is cleaned a second time in boiling piranha. Note that adding a second vertical diamond etching step immediately prior to the quasi-isotropic etch is expected to reduce the necessary undercut time, as in the related single-crystal

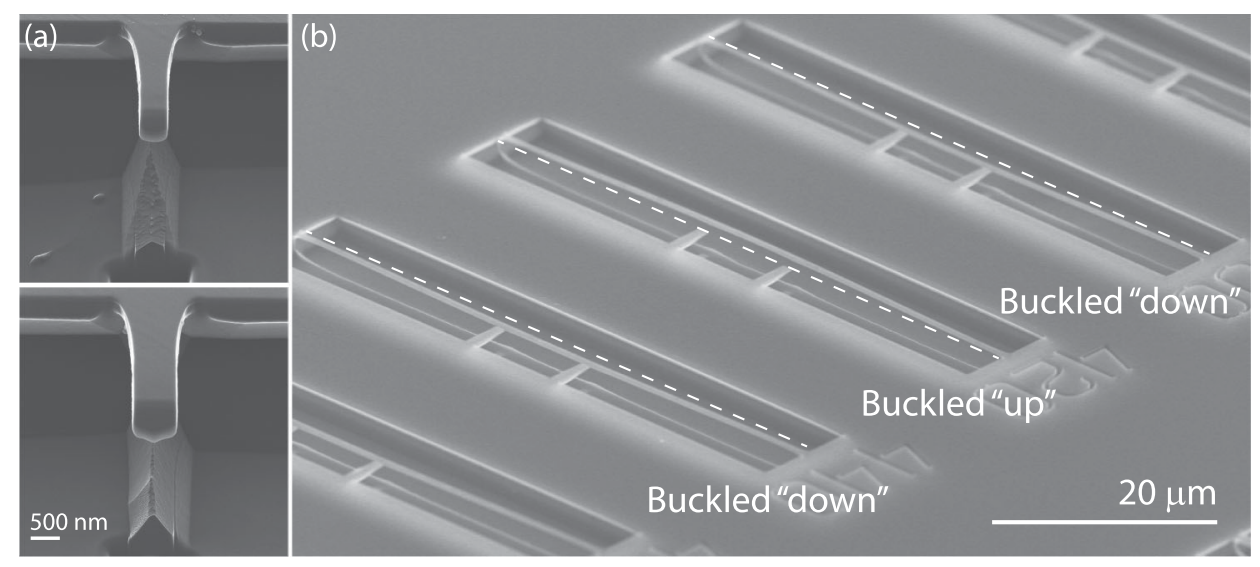

FIG. 6. (a) Nanobeam cross sections, created by focused ion beam (FIB) milling, of two nanobeams with different $w$ patterned on the same chip. Note that the FIB milling process rounds the nanobeam edges, resulting in some apparent curvature in the nanobeam profiles. (b) SEM image of typical nanobeams observed to self-oscillate. Dashed straight lines are guides to emphasize the direction of nanobeam buckling. 
reactive etching and metallization (SCREAM) silicon process [48].

\section{APPENDIX B: MEASUREMENT SETUP}

The optomechanical properties of the diamond nanobeam waveguides are studied by monitoring the optical transmission of a dimpled optical fiber taper positioned in the near field of devices of interest. The dimpled fiber taper is fabricated by modifying the procedure presented in Ref. [50] to use a ceramic mold for creating a dimple. Measurements performed in ambient conditions use high-resolution $(50 \mathrm{~nm})$ dc stepper motors to position the fiber taper. Vacuum (room temperature) and cryogenic measurements are performed in a closed-cycle cryostat (Montana Systems Nanoscale Workstation) whose sample chamber is configured with stick-slip and piezo-positioning stages (Attocube) for controlling the sample and fiber taper positions. Before cooling, a turbo pump is used to evacuate the chamber to pressures in the $10^{-5}$ Torr range. Room-temperature vacuum measurements are performed in these conditions. At $5 \mathrm{~K}$, cryopumping reduces the chamber pressure to the $10^{-6}$ Torr range. During the low-temperature measurements, the fiber taper is positioned in contact with lithographically defined supports on the diamond chip to reduce coupling of vibration from the cryostat cooling stages to low-frequency resonances of the optical fiber taper. These supports, visible in Fig. 6(c), allow the fiber taper to be positioned in the nanobeam near field without contacting the nanobeam.

Two external cavity tunable diode lasers (New Focus Velocity 6700) are used to probe the fiber taper transmission $T$ over a wavelength range from 1475 to $1625 \mathrm{~nm}$. A New Focus 1811 photodetector (PD1) with a noise equivalent power $s_{P}^{(\mathrm{det})}=\sqrt{S_{P}^{(\mathrm{det})}}=2.5 \mathrm{pW} / \sqrt{\mathrm{Hz}}$ is used to monitor the average $\left(\bar{P}_{d}\right)$ and fluctuating $\left(\delta P_{d}(t)\right)$ output power from the fiber taper. A New Focus 1623 detector (PD2) is also used in some measurements of $\bar{P}_{d}$.

A Tektronix RSA5106A real-time spectrum analyzer (RSA) allows fast spectral analysis during the experiments and recording of in-phase and quadrature (IQ) time series of the PD1 output voltage $V(t)$. All of the $S_{v}(f)$ data we present here are generated in postprocessing from $V(t)$ data. By choosing a low center (demodulation) frequency (typically 0 or $2.5 \mathrm{MHz}$ ), and sampling $V(t)$ with a bandwidth exceeding the nanobeam resonance frequencies (typically $5 \mathrm{MHz}$ ), both $\delta T(t)$ and $\bar{T}$ can be recorded by the RSA. To avoid damaging the RSA with a large dc input, a pair of bias tees (Minicircuits ZFBT-6GWB+) together with electrical attenuators are used to reduce the lowfrequency $(<100 \mathrm{kHz})$ components of $V(t)$. The selfoscillation data in Fig. 5 is then acquired in a single $45 \mathrm{~s}$ time series while the fiber height above the sample is stepped in $30 \mathrm{~nm}$ increments. Attenuation of the lowfrequency signal is compensated for in postprocessing.

\section{APPENDIX C: WAVEGUIDE COUPLED-MODE THEORY}

Numerical prediction of the coupling coefficient $\kappa(h)$ describing the interaction between the nanobeam and fiber taper waveguide modes can be obtained from the optical dispersion of the eigenmodes of the coupled waveguides ("supermodes"). This process is described below.

The field propagating through the coupled waveguide system can be represented as a superposition of modes of the uncoupled optical fiber taper and diamond nanobeam. In the case of waveguides with two nearly phase-matched copropagating (positive group velocity) modes, this evolution can be approximately described by coupled-mode equations describing the field amplitude $a_{f, n}(z)$ in the fiber and nanobeam waveguides, respectively, as a function of propagation distance $z$ through the coupling region:

$$
\begin{aligned}
& \frac{d a_{f}}{d z}=-j\left(\beta_{f}+\kappa_{f f}\right) a_{f}-j \kappa a_{n}, \\
& \frac{d a_{n}}{d z}=-j\left(\beta_{n}+\kappa_{n n}\right) a_{n}-j \kappa a_{f},
\end{aligned}
$$

where $\beta_{f}$ and $\beta_{n}$ are the $\lambda$-dependent propagation constants of the uncoupled fiber and nanobeam modes, respectively. Also included in this model are "self-term" corrections $\kappa_{f f, n n}(h)$ to $\beta_{f, n}$ resulting from the modification of the local dielectric environment by the coupled waveguides. The coupler response can also be predicted by solving Eqs. (C1) and $(\mathrm{C} 2)$ with boundary condition corresponding to unity input power to the fiber taper $\left(\left|a_{f}(0)\right|^{2}=1,\left|a_{n}(0)\right|^{2}=0\right)$, resulting in the taper transmission $T(\lambda, h)$ given by Eq. (1) in the main text.

Numerically, prediction of $\kappa(h)$ and $\kappa_{a a, b b}(h)$ can be realized from the dispersion of the supermodes of the coupled waveguides. These modes can be modeled as superpositions of the uncoupled modes of waveguide $a$ and $b$. Their $z$ dependence is of the form $e^{-i \beta_{ \pm} z}$, which satisfies Eqs. (C1) and (C2) for

$$
\beta_{ \pm}=\frac{\tilde{\beta}_{f}+\tilde{\beta}_{n}}{2} \pm \sqrt{\left(\frac{\tilde{\beta}_{f}-\tilde{\beta}_{n}}{2}\right)^{2}+\kappa(h)^{2}},
$$

with $\tilde{\beta}_{f, n}=\beta_{f, n}(\lambda)+\kappa_{f f, n n}(h)$ [51].

Intrawaveguide and self-coupling coefficients $\kappa(h)$ and $\kappa_{f f, n n}(h)$ are determined by fitting $\beta_{ \pm}(h, \lambda)$ with Eq. (C3). $\beta_{ \pm}(h, \lambda)$ of the coupled waveguides and $\beta_{f, n}(\lambda)$ of the uncoupled waveguides are calculated numerically with a mode solver (Lumerical MODE Solutions) and are shown in Figs. 1(c) and 7. The resulting values for $\kappa(h)$ and $\kappa_{f f, n n}(h)$, shown in Fig. 8, are found to decay exponentially with $h$. For simplicity, it is assumed that $\kappa_{f f}=\kappa_{n n}$ during the fitting process, and that $\lambda$ dependence is assumed to be small over the coupling bandwidth of interest. Note that 


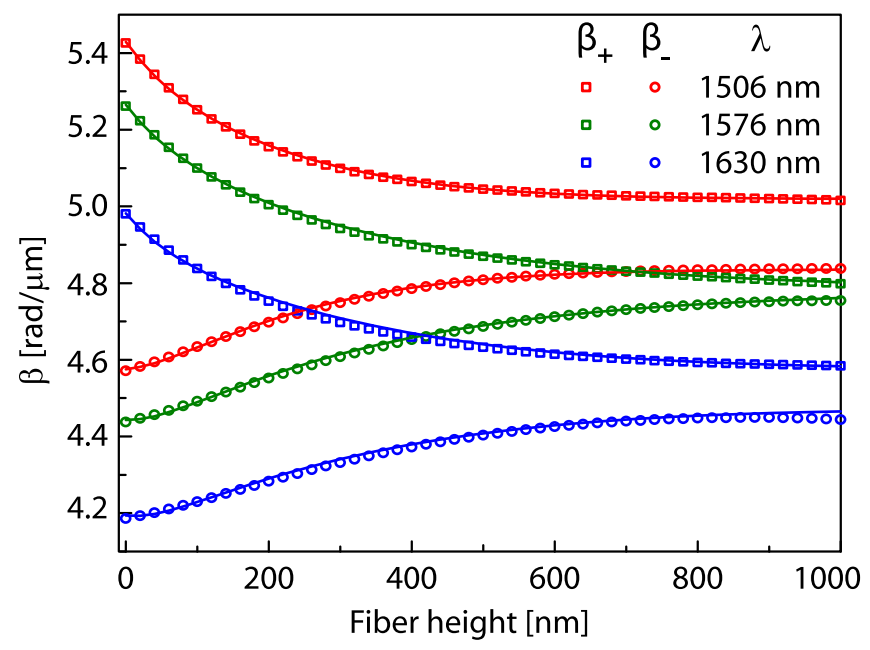

FIG. 7. Propagation constants $\left(\beta_{ \pm}\right)$of the even and odd supermodes of the coupled nanobeam and fiber taper waveguides, as a function of waveguide separation $h$, for varying $\lambda$. Points indicated by open squares and circles where calculated using Lumerical MODE Solutions. Solid lines are fits using the coupled mode theory model described in Appendix C.

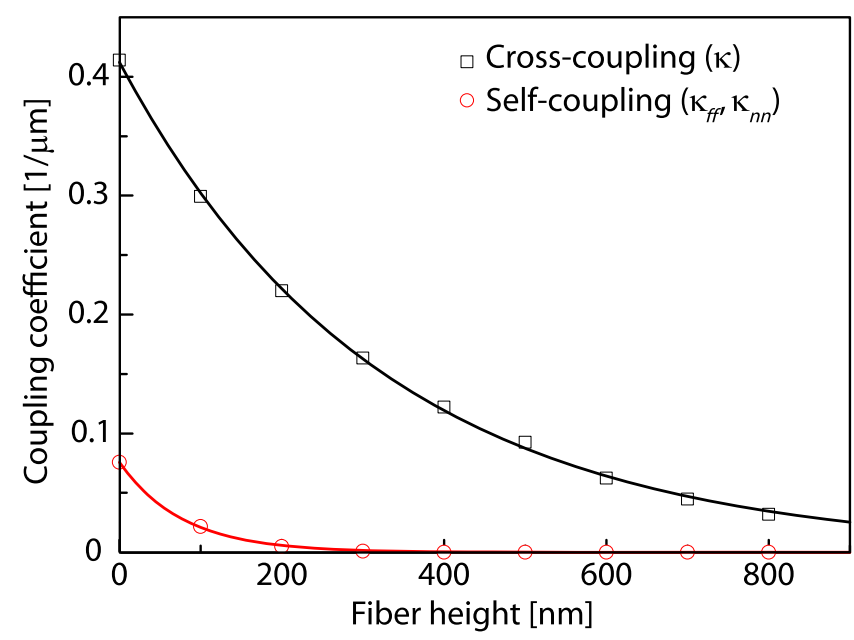

FIG. 8. Waveguide coupling coefficients as a function of fiber taper and nanobeam waveguide separation $h$. Each data point is obtained by fitting $\beta_{ \pm}(\lambda)$ with the coupled-mode theory model. Solid lines are single exponential fits to the data points.

while $\kappa(h)$ decays over a length-scale comparable to the waveguide evanescent field decay, $\kappa_{f f, n n}(h)$ are near-field terms that decay much more quickly and do not significantly impact the predicted values of $\kappa(h)$ for $h>100 \mathrm{~nm}$.

For a given $h, \beta_{ \pm}(\lambda)$ can be converted to an effective index dispersion curve, $n_{ \pm}(\lambda)=\beta_{ \pm}(\lambda) \lambda / 2 \pi$, as shown in Fig. 1(c), which clearly exhibits an anticrossing near $\beta_{f}=$ $\beta_{n}$ whose width scales with $|\kappa|$. The excellent agreement between the numerically calculated $\beta_{ \pm}$and the semianalytic model described by Eq. (C3) indicates that this model is suitable for predicting the waveguide coupling.

\section{APPENDIX D: OPTOMECHANICAL COUPLING AND BACKACTION}

A cavity optomechanical system with both dissipative and dispersive optomechanical coupling can be realized by incorporating the fiber-coupled diamond nanobeam within an optical resonator. Although not demonstrated in this paper, such a system could be achieved by either terminating the nanobeam waveguide with photonic crystal mirrors or fabricating a racetrack structure incorporating a nanobeam along each side. Both approaches require further development of the fabrication process. In this appendix, dissipative and dispersive coupling coefficients are derived for such a system, and the potential for using this system for optomechanical cooling is analyzed.

\section{Dissipative optomechanical coupling coefficient}

The rate of "external" coupling of light between a nanobeam and the forward propagating mode of a fiber taper waveguide $\gamma_{e}$ can be determined from $\bar{T}$ and the round-trip time of light circulating in the hypothetical cavity in which the nanobeam is integrated. A standingwave cavity formed from a nanobeam terminated by end mirrors couples bidirectionally to the fiber taper, as light passes through the coupling region twice per round-trip: once propagating in the forward direction and once propagating in the backward direction. In this system,

$$
\gamma_{e}=\frac{v_{g}}{2 L} \ln (1 / \bar{T}) \sim \frac{v_{g}}{2 L} \frac{1-\bar{T}}{\sqrt{\bar{T}}},
$$

where $L$ is the total nanobeam length, $v_{g}$ is the group velocity of light in the nanobeam, and the right-hand expression is a good approximation when $\bar{T} \sim 1$ [75]. The "parasitic" coupling rate into the backward propagating fiber taper mode is $\gamma_{p}=\gamma_{e}$, and the total output coupling rate is $2 \gamma_{e}$. Parasitic loss can limit the effectiveness of dissipative optomechanical cooling, as discussed below.

The corresponding dissipative optomechanical coupling coefficient into the forward propagating fiber taper mode is given by

$$
g_{\gamma}^{(e)}=\frac{\partial \gamma_{e}}{\partial h} \chi \sim-\frac{v_{g}}{2 L} \frac{1}{\sqrt{\bar{T}}} \frac{1+\bar{T}}{2 \bar{T}} \frac{\partial \bar{T}}{\partial h} \chi .
$$

Here, $0 \leq \chi \leq 1$ is a dimensionless parameter accounting for imperfect correspondence between nanobeam displacement and change in waveguide separation $h$; i.e., $\chi$ is the average value of $d h / d x$ across the coupler region for the mechanical resonance of interest. The parasitic dissipative coupling coefficient to the backward propagating fiber mode for a standing-wave cavity is $g_{\gamma}^{(p)}=g_{\gamma}^{(e)}$.

A nanobeam integrated within a traveling-wave cavity, e.g., a racetrack resonator, can be coupled unidirectionally to the fiber taper, assuming that backscattering within the resonator is small compared to internal loss. In this 
configuration light passes through the coupling region once per round-trip, $2 L$ represents the total round-trip path length, and both $\gamma_{p}=0$ and $g_{\gamma}^{(p)}=0$, while $g_{\gamma}^{(e)}$ is unchanged from the standing-wave configuration.

\section{Dispersive optomechanical coupling coefficient}

The dispersive optomechanical coupling coefficient $g_{\omega}$ is determined by the $h$ dependence of the round-trip phase $\phi(h)$ accumulated by light circulating in the nanobeam. In general, $\phi$ depends on the waveguide mode mixing within the coupling region. However, at phase-matching it can be shown that the $h$ dependence of $\phi$ arises solely from $\kappa_{n n}$ and $\kappa_{f f}$ discussed in Appendix C. For a standing-wave cavity geometry,

$$
\frac{\phi}{2}=\beta_{o} L+\frac{\kappa_{f f}+\kappa_{n n}}{2} L_{c} .
$$

The contributions to $\phi$ from $\kappa_{f f}$ and $\kappa_{n n}$ are due to a renormalization of the dielectric environment of each waveguide due to its neighbor. The corresponding dispersive optomechanical coupling coefficient is given by

$$
g_{\omega}=\frac{v_{g}}{2 L} \frac{\partial \phi}{\partial h} \chi=v_{g} \frac{L_{c}}{L} \frac{\partial}{\partial h}\left(\frac{\kappa_{f f}+\kappa_{n n}}{2}\right) \chi .
$$

Note that for $\kappa^{2} \gg\left(\kappa_{f f}-\kappa_{n n}\right)^{2}$, we can use Eq. (C3) to write

$$
g_{\omega}=\frac{\omega}{n_{g}} \frac{L_{c}}{L} \frac{\partial}{\partial h}\left(\frac{n_{+}+n_{-}}{2}\right) \chi,
$$

where $n_{g}=c / v_{g}$. This expression provides a convenient means to calculate $g_{\omega}$ from $n_{ \pm}(h)$ of the coupled waveguide supermodes at phase-matching. As discussed in Appendix F, Eq. (D5) also shows that $g_{\omega}$ is proportional to the optical gradient force, as in standard cavity optomechanical systems [53].

Equations (D3)-(D5) can be modified for the case of a traveling-wave cavity, where circulating photons interact with the coupling region once per round-trip, by changing $L_{c} \rightarrow L_{c} / 2$.

\section{Predicted optomechanical coupling}

The optomechanical coupling coefficients of the system studied in the main text can be predicted from Eqs. (D2) and (D5) using measured values of $\bar{T}(h)$ and simulated $n_{ \pm}(h)$. Figure 9(a) shows $g_{\gamma}^{(e)}(h), g_{\omega}(h)$, and $\gamma_{e}(h)$ predicted for the device with the fit of $\bar{T}(h)$ used in Fig. 4(b), assuming $L=L_{c}=7 \mu \mathrm{m}$ and $\chi=1.0$. This shows that $g_{\omega}$ is small compared to $g_{\gamma}^{(e)}$ for $h>200 \mathrm{~nm}$. For the device geometry we study here, dispersive coupling is predicted to vanish at a critical value of $h \sim 450 \mathrm{~nm}$. The maximum dissipative coupling into the forward propagating fiber mode is $g_{\gamma}^{(e)} / 2 \pi=45 \mathrm{GHz} / \mathrm{nm}$.
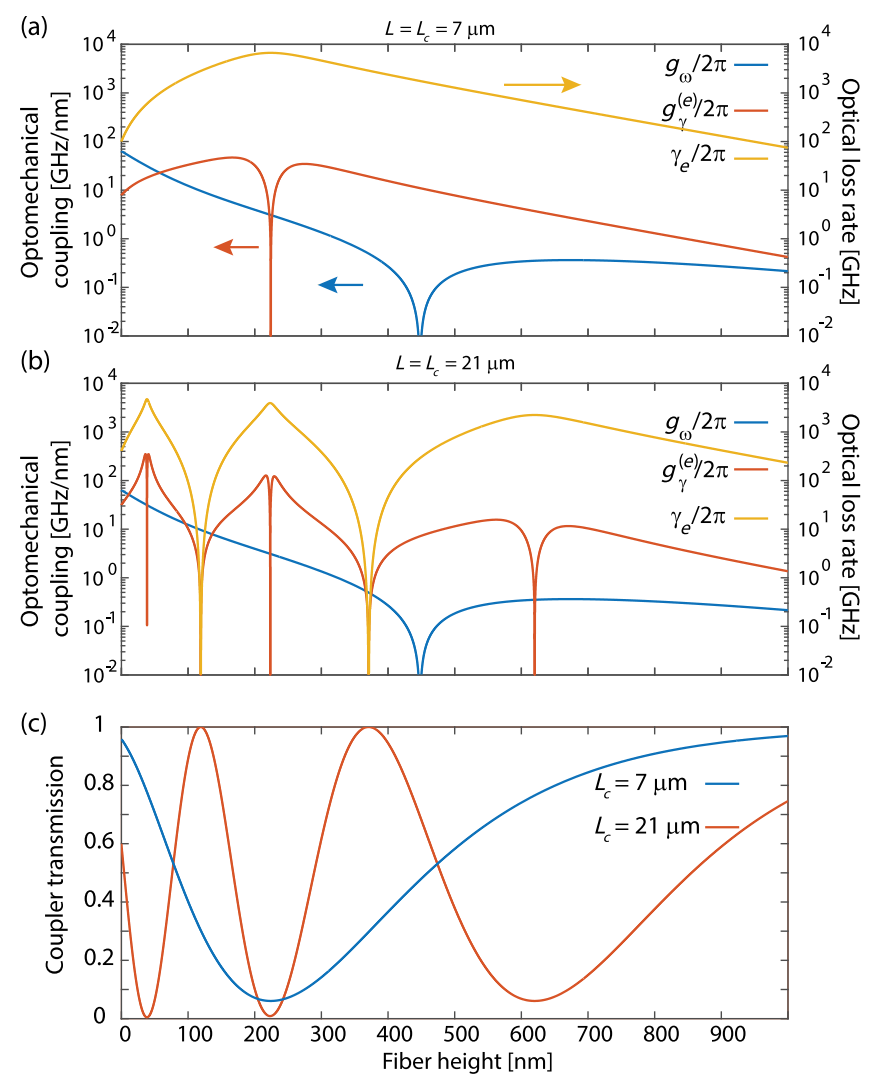

FIG. 9. Predicted optomechanical coupling coefficients for varying fiber height when (a) $L_{c}=7 \mu \mathrm{m}$, as in the experiment, and (b) $L_{c}=21 \mu \mathrm{m}$, for a nanobeam of length $L=L_{c}$. Also shown is the coupling rate between the nanobeam and the forward propagating fiber taper waveguide mode $\gamma_{e}(h)$. (c) Corresponding transmission functions for $L_{c}=7 \mu \mathrm{m}$ and $L_{c}=21 \mu \mathrm{m}$.

For cavity-optomechanics applications, it is desirable to operate in a regime of small $\gamma_{e}$ and large $g_{\gamma}^{(e)}$. This may be achieved in future studies by increasing $L_{c}$. Experimentally, this requires modifying the fiber taper dimple profile or using a straight fiber taper as in Ref. [52]. Figure 9(b) shows the predicted optomechanical coupling when $L_{c}=$ $L=21 \mu \mathrm{m}$, assuming the same $\kappa(h)$ and slightly imperfect phase-matching extracted from fits to the experimental data used in Fig. 4(b). The corresponding simulated $\bar{T}(h)$ is shown in Fig. 9(c). For this system, low-loss and $g_{\gamma}^{(e)} \gg g_{\omega}$ are possible, for example, near $h \sim 370 \mathrm{~nm}$.

\section{Considerations for dissipative optomechanical cooling using a cavity}

Dissipative optomechanical coupling has been shown theoretically [64] and experimentally [63] to allow cooling of a mechanical resonator in an unresolved sideband cavityoptomechanical system. Unresolved sideband cooling requires tuning of the relative strength of $g_{\gamma}^{(e)}, g_{\omega}$, and $\gamma_{e}$. This is possible in the system studied here by adjusting $h$, 
as shown in Figs. 9(a) and 9(b). However, dissipative optomechanical cooling performance is limited by optical loss $\gamma_{i}+\gamma_{p}$ into channels other than the input or output channel, and cooling to the quantum ground state requires that $\gamma_{e} \gg \gamma_{i}, \gamma_{p}$ [64].

Waveguide absorption, scattering loss, and leaky mirrors typically determine $\gamma_{i}$ in nanophotonic cavities; these losses are $<10 \mathrm{GHz}$ in state-of-the-art diamond devices [47]. Eliminating $\gamma_{p}$ is possible through development of traveling-wave optical cavity devices incorporating the diamond nanobeam, such as an oval racetrack resonator [47]. Note that such cavities would additionally require low backscattering rates to satisfy this criteria. Alternatively, implementing dissipative waveguide coupling between on-chip waveguides may allow replacing the fiber taper waveguide with a "single-sided" input-output coupling waveguide, as in Ref. [72], preventing leakage into a parasitic backwards propagating waveguide mode at the expense of the tunable nature of the coupling demonstrated here.

To evaluate the potential for dissipative optomechanical cooling using the demonstrated interface integrated within a cavity, we use the formalism in Refs. [64]. We assume that the nanobeam discussed above is incorporated into one half of an oval racetrack optical resonator with sides of length $L_{c}=21 \mu \mathrm{m}$ and semicircular ends of radius $10 \mu \mathrm{m}$, and is unidirectionally coupled to the fiber taper with no backscattering. Figure 10 shows the predicted minimum phonon number $n$ achievable as a function of $h$, assuming $\gamma_{i} / 2 \pi=1 \mathrm{GHz}$ and $\gamma_{p}=0, P_{i}=2 \mathrm{~mW}$ of power input to the fiber taper, and a bath temperature of $5 \mathrm{~K}$. Other device parameters are given in the figure caption. For this system,

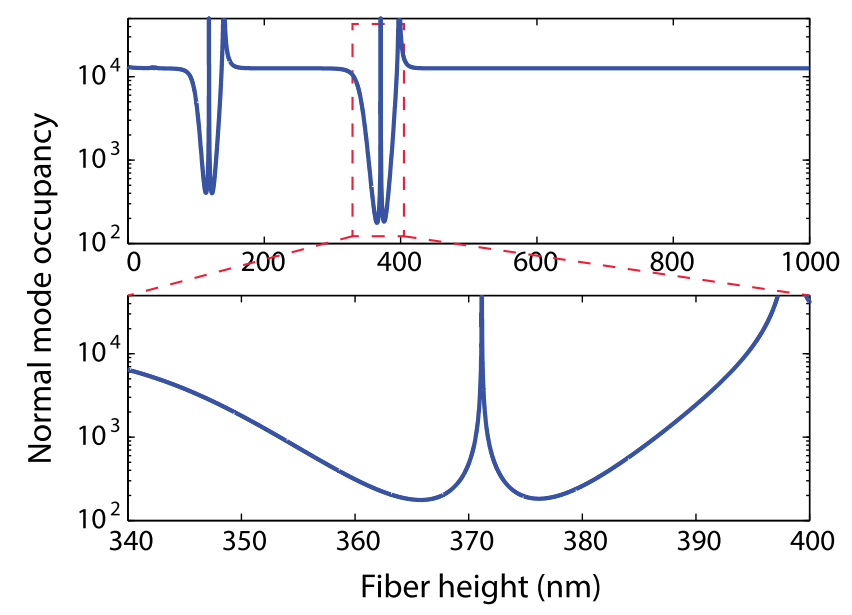

FIG. 10. Predicted achievable phonon number via optomechanical cooling of a fiber taper coupled nanobeam incorporated into an oval racetrack resonator $\left(\gamma_{p}=0\right)$. Device parameters are $\gamma_{i} / 2 \pi=1 \mathrm{GHz}, L_{c}=L=21 \mu \mathrm{m}, \omega_{m} / 2 \pi=7.3 \mathrm{MHz}, m=3.1 \mathrm{pg}$, $Q_{m}=10^{6}, 5 \mathrm{~K}$ bath temperature. For the oval racetrack cavity, $2 L=2 L_{c}+2 \pi R_{c}$, where $R_{c}=10 \mu \mathrm{m}$ is the radius of curvature of the cavity end. dissipative optomechanical cooling from a phonon occupancy of $n_{\mathrm{ph}} \sim 15000$ to $n_{\mathrm{ph}} \sim 200$ is predicted when the fiber taper is positioned on either side of the transmission "recovery" point $h \sim 370 \mathrm{~nm}$, where $\bar{T} \rightarrow 1$. Reducing $\gamma_{i} / 2 \pi$ to $0.1 \mathrm{GHz}$, a technically challenging but potentially attainable value, would allow $n=16$ to be reached. For extremely small $\gamma_{i} / 2 \pi=1 \mathrm{MHz}, n<1$ is possible. These results highlight the key role of $\gamma_{i}$, and also show that for the purposes of optomechanical cooling, operating the waveguide coupler near maximum $g_{\gamma}^{(e)}$ is not optimal due to accompanying large $\gamma_{e}$.

This calculation is performed using $n_{\mathrm{ph}}=\left(n_{\mathrm{th}} \gamma_{m}+\right.$ $\left.n_{\mathrm{ba}} \gamma_{\mathrm{om}}\right) /\left(\gamma_{m}+\gamma_{\mathrm{om}}\right)$, where $n_{\text {th }}$ is the thermal phonon occupancy, $n_{\mathrm{ba}}=S_{F F}\left(-\omega_{m}\right) /\left[S_{F F}\left(\omega_{m}\right)-S_{F F}\left(-\omega_{m}\right)\right]$ is the phonon population created by optomechanical backaction, and $\gamma_{\mathrm{om}}=x_{\mathrm{zpf}}^{2}\left[S_{F F}\left(\omega_{m}\right)-S_{F F}\left(-\omega_{m}\right)\right]$ is the optomechanical damping rate. $x_{\mathrm{zpf}}$ is the zero-point fluctuation amplitude of the resonator, and $S_{F F}(\omega)$ is the total optomechanical force power spectral density acting on the nanobeam, given in Ref. [64] as

$$
\begin{aligned}
S_{F F}(\omega)= & \frac{\tilde{B}^{2} N}{4 x_{\mathrm{zpf}}^{2}}\left(\gamma_{e} \frac{\left[\omega+2 \Delta-2 \gamma_{t} \frac{\tilde{A}}{\tilde{\tilde{B}}}\right]^{2}}{(\omega+\Delta)^{2}+\gamma_{t}^{2} / 4}\right. \\
& \left.+\left(\gamma_{i}+\gamma_{p}\right) \frac{\left[\left(\Delta-2 \frac{\tilde{A}}{\tilde{\tilde{B}}} \gamma_{t}\right)^{2}+\frac{\gamma_{t}^{2}}{4}\right]}{(\omega+\Delta)^{2}+\gamma_{t}^{2} / 4}\right) .
\end{aligned}
$$

Here, $N(h)$ is the intracavity photon number, $\tilde{B}(h)=$ $g_{\gamma}^{(e)} x_{\mathrm{zpf}} / \gamma_{e}$ and $\tilde{A}(h)=g_{\omega} x_{\mathrm{zpf}} / \gamma_{e}$ are the normalized optomechanical coupling coefficients, and $\gamma_{t}=\gamma_{e}+\gamma_{p}+\gamma_{i}$. For the calculations shown here, we set the detuning between the cavity mode and input power to $\Delta=\Delta_{\text {opt }}=\omega_{m} / 2+\tilde{A} / \tilde{B} \gamma_{t}$, which causes cancellation between the dissipative and dispersive backaction at the input port of the cavity.

\section{APPENDIX E: OPTOMECHANICAL TRANSDUCTION SENSITIVITY}

This appendix describes the procedure for predicting the theoretical measurement sensitivity of the optomechanical waveguide read-out and for extracting the experimentally observed measurement sensitivity from measured thermomechanical signals. Single-sided power spectral densities are used throughout.

\section{Theoretical sensitivity}

The displacement sensitivity of the waveguideoptomechanical system is determined by the minimum mechanical motion to actuate a signal larger than the noise floor of the measurement apparatus. For direct photodetection of the optical power transmitted by the waveguide, the power spectral density $S_{v}^{(s)}(f)$ of the transduced 
signal from a mechanical displacement described by spectral density $S_{x}(f)$ is

$$
S_{v}^{(s)}(f)=S_{x}(f)\left(g_{\mathrm{ti}} P_{d} \frac{\partial T}{\partial x}\right)^{2},
$$

where $g_{\mathrm{ti}}$ is the detector transimpedance gain, $P_{d}$ is the detected power in the absence of coupling $(T=1)$, and $|\partial T / \partial x|$ describes the optomechanical actuation of the coupler. Similarly, the measurement noise can be written as

$$
S_{v}^{(n)}(f)=S_{v}^{(\mathrm{SN})}(f)+S_{v}^{(\mathrm{det})}(f) .
$$

$S_{v}^{(\text {det })}$ describes noise intrinsic to the detector and is related to the detector's noise equivalent power figure $\left(S_{P}^{(\mathrm{det})}\right)$ by $S_{v}^{(\mathrm{det})}=g_{\mathrm{ti}}^{2} S_{P}^{(\mathrm{det})}$. The contribution from photon shot noise is given by

$$
S_{v}^{(\mathrm{SN})}(f)=g_{\mathrm{ti}}^{2} \frac{2 \hbar \omega_{o} T P_{d}}{\eta_{\mathrm{qe}}},
$$

where $\eta_{\mathrm{qe}}$ is the detector quantum efficiency. Note that the impact of shot noise is affected by the operating point $T$ of the coupler. This analysis does not consider optomechanical backaction, which is small for the optical powers used in the high-sensitivity measurements presented here.

To calculate the minimum detectable spectral density $S_{x}^{o}$, we require unity signal-to-noise ratio, $S_{v}^{(s)}=S_{v}^{(n)}$, resulting in

$$
S_{x}^{o}=\frac{2 \hbar \omega_{o} T P_{d} / \eta_{\mathrm{qe}}+S_{P}^{(\mathrm{det})}}{\left(P_{d} \frac{\partial T}{\partial x}\right)^{2}} .
$$

$S_{x}^{o}$ has units of $\mathrm{nm}^{2} / \mathrm{Hz}$ and is related to the minimum detection sensitivity given in the text by $s_{x}^{o}=\sqrt{S_{x}^{o}}$. In the case of a codirectional evanescent coupler, $\partial T / \partial x$ can be calculated from Eq. (1) in the main text. For perfect phasematching $(\Delta \beta=0)$,

$$
\frac{\partial T}{\partial x}=-\sin (2 \kappa L) \frac{\partial \kappa}{\partial h} \frac{\partial h}{\partial x} .
$$

Alternately, $\partial T / \partial x$ can be measured experimentally by recording $T(h)$ and determining $d T / d h$. This approach is used together with Eq. (E4) to generate the predicted measurement sensitivity in Fig. 4, assuming that $\partial h / \partial x=-1$.

\section{Thermomechanical calibration}

The observed thermal nanobeam motion is used to calibrate the measurement noise floor using a standard procedure described in, for example, Refs. [61,76]. Thermomechanical resonances in $S_{v}(\omega)$ are fit using $S_{v}(\omega)=S_{v}^{(n)}+G S_{x}^{(\text {th })}(\omega)$, where $S_{x}^{(\text {th })}(\omega)$ is the singlesided power spectral density of a thermal oscillator,
$S_{x}^{(\mathrm{th})}(\omega)=\frac{4 k_{B} T_{e} \omega_{m}}{Q_{m}} \frac{1}{m\left[\left(\omega-\omega_{m}^{2}\right)^{2}+\left(\omega \omega_{m} / Q_{m}\right)^{2}\right]}$,

and $G$ is a constant determined by the transduction gain of the measurement, as described theoretically by Eq. (E1), and treated as a fitting parameter for the purpose of the calibration procedure. Here, $k_{B}$ is Boltzmann's constant, $T_{e}$ is the operating temperature, and $m$ is the effective mass of the resonance, as defined in Ref. [77]. From the fit values, spectra can be converted from electrical $(\mathrm{W} / \mathrm{Hz})$ to displacement $\left(\mathrm{m}^{2} / \mathrm{Hz}\right)$ power spectral density: $S_{x}=S_{v} / G$.

\section{APPENDIX F: OPTICAL GRADIENT FORCE}

Dielectric objects in an evanescent field experience an optical gradient force. The optical gradient force between coupled waveguides can be predicted from $n_{ \pm}(h)$ using the formalism of $\mathrm{Ma}$ and Povinelli [78]. Following this formalism, the force induced by power $P_{ \pm}$in each of the supermodes is given by

$$
F_{ \pm}=\left.\frac{P_{ \pm} L_{c}}{c} \frac{\partial n_{ \pm}}{\partial h}\right|_{\omega_{o}}=-\left.\frac{P_{ \pm} L_{c}}{c} \frac{\partial n_{ \pm}}{\partial x}\right|_{\omega_{o}} .
$$

Operating at phase-matching with power $P_{i}$ input into the fiber taper, the power in the even and odd supermodes is $P_{ \pm}=P_{i} / 2$, and the total optical gradient force on the nanobeam is

$$
F_{g}=\frac{P_{i} L_{c}}{2 c}\left(\frac{\partial n_{+}}{\partial h}+\frac{\partial n_{-}}{\partial h}\right),
$$

where positive (negative) $F_{g}$ indicates a repulsive (attractive) force. Figure 11 shows the predicted $F_{g}(h) / L_{c} P_{i}$ for varying alignment of the fiber taper with the center axis of the nanobeam. Corrections due to the curvature of the

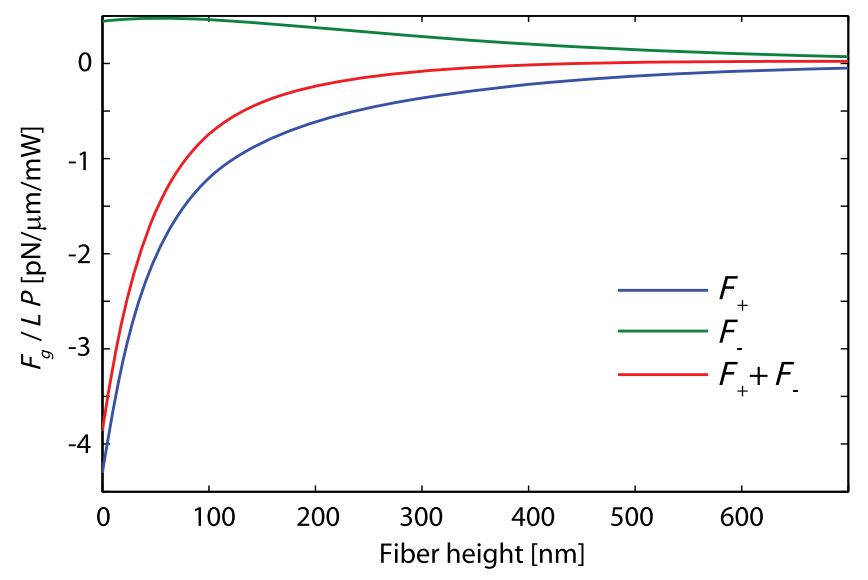

FIG. 11. Optical gradient force as a function of $h$, when the fiber taper is aligned along the nanobeam axis, and offset $500 \mathrm{~nm}$ laterally, approximating the position in the self-oscillation measurements. 
dimpled fiber taper are not considered explicitly and are assumed to be accounted for by the effective coupler length $L_{c}$ extracted from the experimental measurements. Given this approximation, $F_{g}$ is constant over the interaction length of the ideal two-port waveguide coupler and vanishes outside of the coupling region.

\section{APPENDIX G: NANOBEAM PHOTOTHERMAL AND NONLINEAR DYNAMICS}

Waveguide-optomechanical coupling is observed to induce nanobeam self-oscillations, as shown in Fig. 5 of the main text. Here, we analyze this effect by modeling the nanobeam as a nonlinear harmonic oscillator interacting with the optical field of the coupled waveguides through a dynamic photothermal force and an instantaneous optical gradient force $[1,70,79,80]$.

\section{Nanobeam equation of motion}

The dynamics of the nanobeam resonance driven by a stochastic thermal force $F_{s}(t)$ and coupled to the optical fiber taper are approximately described by the following equation of motion:

$$
\begin{array}{r}
\ddot{x}+\frac{\omega_{m}}{Q_{m}} \dot{x}+\left(\omega_{m}^{2}(\bar{x})+\alpha_{3} x^{2}+\alpha_{2} x\right) x \\
=\frac{1}{m}\left(F_{g}(x)+F_{\mathrm{pt}}(x, t)+F_{s}(t)\right),
\end{array}
$$

where $x(t)$ is the displacement amplitude of the nanobeam mechanical resonance of interest, defined relative to the position of the undriven nanobeam. Changes in $x$ modulate the nanobeam and the fiber taper spacing $h$, and for the case of the $\mathbf{v}_{1}$ resonance, $h(x)=h_{o}-x(t)$ if the fiber taper is positioned $h_{o}$ above the center of the nanobeam. Optomechanical coupling arises from the dependence of the optical gradient force, $F_{g}(h(x))$ and the photothermal force $F_{\mathrm{pt}}(h(x))$ on nanobeam position. In addition, $\omega_{m}$ varies due to internal strain resulting from optically induced changes in static deflection $\bar{x}$ and local nanobeam temperature relative to the environment $\Theta$. For sufficiently large $|x(t)|$, the nanobeam response becomes nonlinear, as described by $\alpha_{2}$ and $\alpha_{3}$.

\section{Thermal effects}

Local heating of the nanobeam by waveguide optical absorption occurs on a time scale determined by the nanobeam geometry and material properties. The thermal dynamics of the nanobeam, defined by the maximum temperature change $\Theta$, relative to the operating temperature, are assumed to follow

$$
\frac{d \Theta}{d t}=-\kappa_{t} \Theta+R L_{i} \zeta P_{n},
$$

where $\tau=1 / \kappa_{t}$ is the nanobeam thermal time-constant and $P_{n}$ is the optical power coupled into the nanobeam. In the absence of delayed optical feedback (i.e., a cavity), $P_{n}$ instantaneously follows $x$ and is given by

$$
P_{n}=[1-T(h(x))] P_{i},
$$

with $T(h)$ described by Eq. (1) in the main text. Here, $\zeta$ is the per unit length absorption coefficient of the nanobeam waveguide and $R$ is the heating power per unit of absorbed optical power. Ideally, $\zeta$ is determined by the material properties of diamond, but in nanophotonic devices it can be modified by imperfect surfaces. The effective nanobeam waveguide optical interaction length is given by $L_{i}(h)=\int_{0}^{L}\left|a_{n}(z)\right|^{2} d z /\left|a_{f}(0)\right|^{2}$ and represents the distance over which light propagates in the nanobeam. It can be calculated from Eqs. (C1) and (C2).

Local heating of nanobeam waveguides induces deflections in the nanobeam position. We write the corresponding photothermal force as

$$
F_{\mathrm{pt}}(x, t)=\mathcal{F} \Theta(x, t) \frac{\kappa_{t}}{R},
$$

where $\mathcal{F}$ is the force per unit absorbed power $\left(L_{i} \zeta P_{n}\right)$ in steady state. The resulting change in nanobeam deflection is $\delta \bar{x}_{\mathrm{pt}}=F_{\mathrm{pt}} / k$, where $k=\omega_{m}^{2} m$ is the nanobeam spring constant.

Local heating and accompanying thermal expansion of the device also modifies $\omega_{m}$. This effect is represented by

$$
\frac{d \omega_{m}}{d \Theta}=C_{t} \frac{\kappa_{t}}{R}
$$

where $C_{t}$ is a constant related to the elastic properties of the nanobeam and has units $\operatorname{rad~s}^{-1} \mathrm{~W}^{-1}$.

\section{Static response of compressed nanobeams}

In general, $C_{t}$ and $\mathcal{F}$ sensitively depend on both the nanobeam elastic properties and geometry and the internal residual stress acting on the device. The nanobeams used in the self-oscillation studies have significant internal stress, manifesting in smaller $\omega_{m}$ than expected from their nominal dimensions, and can be in a buckled geometry, as shown in the SEM image in Fig. 6(b). To model nanobeam behavior in the presence of compressive stress and buckling, together with imperfect nanobeam shape and clamping points, we consider both an approximate analytic model and finite element simulations.

The behavior of an ideal beam under axial compressive loads has been widely analyzed [81]. For compressive axial load $F_{i}, \omega_{m}$ of a nanobeam with maximum deflection $\bar{x}$ can be expressed as 


$$
\omega_{m}^{2}=\left(\omega_{m}^{i}\right)^{2}\left(1-\frac{F_{i}}{F_{c}}+3 \bar{x}^{2} \frac{A E}{F_{c}} \frac{\pi^{2}}{(2 L)^{2}}\right)
$$

where $\omega_{m}^{i}$ is the resonance frequency of an ideal unloaded nanobeam, $A$ is the nanobeam cross-sectional area, $E$ is Young's modulus, and $F_{c}$ is the critical buckling load. Equation (G6) is equivalent to the model presented in Ref. [71] and reveals the interplay between deflection, axial load, and stiffness. For example, we see that $d \omega_{m} / d \bar{x} \propto \bar{x}$, which is a result of more efficient conversion of transverse actuation to axial strain with increasing nanobeam curvature. Locally heating the nanobeam modifies $F_{i} \rightarrow F_{i}+\eta \epsilon E \Theta$, where $\epsilon$ is the thermal expansion coefficient of the nanobeam and $0<\eta \leq 1$ is a geometric factor that accounts for nonuniform heating distribution of the nanobeam. In general, $F_{i}$ and $\bar{x}$ are not independent. However, in an ideally buckled nanobeam [81], we can show that

$$
\frac{d \bar{x}}{d \Theta}=\frac{\mathcal{F}}{k} \frac{\kappa_{t}}{R}=\bar{x} \frac{\eta \epsilon w d E}{2 m \omega_{m}^{2} L},
$$

indicating that photothermal deflection can be enhanced in buckled nanobeams with $|\bar{x}|>0$.

Although the ideal nanobeam buckling model is instructive, it fails to reproduce experimental features such as deflection for axial load below the critical buckling load and effects related to imperfect elastic clamping points and beam deformation [82]. To predict $\mathcal{F}$ and $C_{t}$ while including nanobeam nonidealities, we use finite element ANSYS software to simulate $\omega_{m}$ and $\bar{x}$ as a function of axial and transverse loads and for specified absorbed power. The results, summarized in Fig. 12 and Table I, indicate that significant enhancement of $\mathcal{F}$ compared to an unloaded nanobeam is expected.

The simulations are conducted as follows. The simulated structure consists of a nanobeam with dimensions $L \times w \times$ $d=80 \times 0.48 \times 0.25 \mu \mathrm{m}^{3}$ and includes the surrounding diamond chip. Clamping-point geometry is found to significantly affect the simulated nanobeam properties. In fabricated nanobeams, the undercut process results in relatively complex clamping-point geometry. Here, the clamping points are modeled with a triangular vertical profile roughly approximating that of fabricated structures, extending $0.5 \mu \mathrm{m}$ on the nanobeam bottom surface and $1.0 \mu \mathrm{m}$ vertically along the undercut sidewall, as shown in Fig. 12(a). $\omega_{m}$ and $\bar{x}$ are then calculated as a function of $F_{i}$, as shown in Fig. 12(b). Agreement between simulated and experimental $\omega_{m}$ is realized at two values of $F_{i}$, corresponding to prebuckled and postbuckled nanobeam states, with $\bar{x}=-9$ and $-123 \mathrm{~nm}$, respectively, where negative $\bar{x}$ indicates buckling down.

$\mathcal{F}$ and $C_{t}$ are estimated by simulating changes to $\bar{x}$ and $\omega_{m}$ when power $P_{\text {abs }}$ is uniformly absorbed across half of the nanobeam. During these simulations the bottom surface (a)
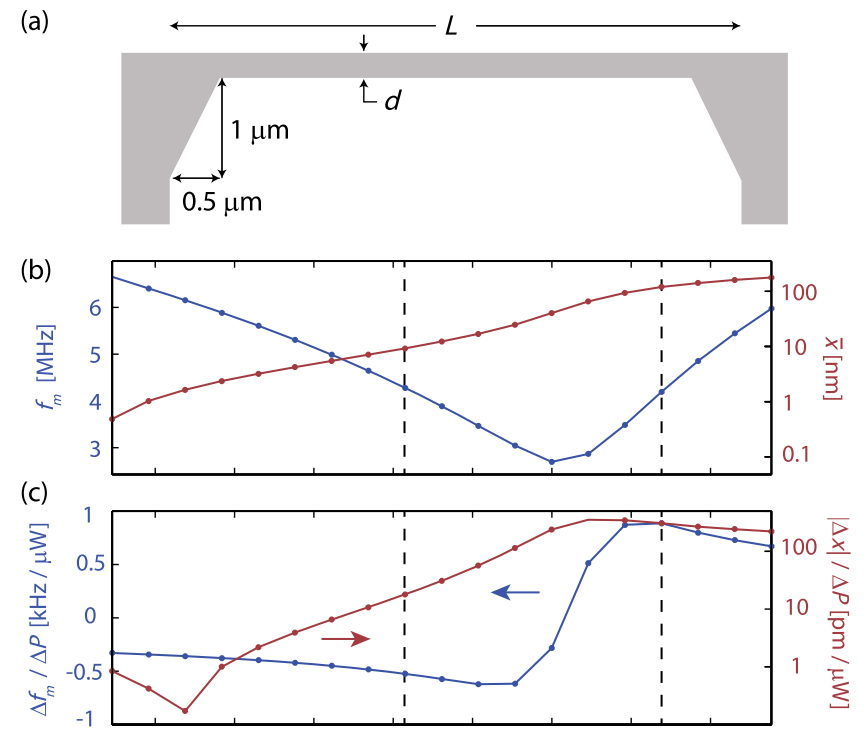

(d)

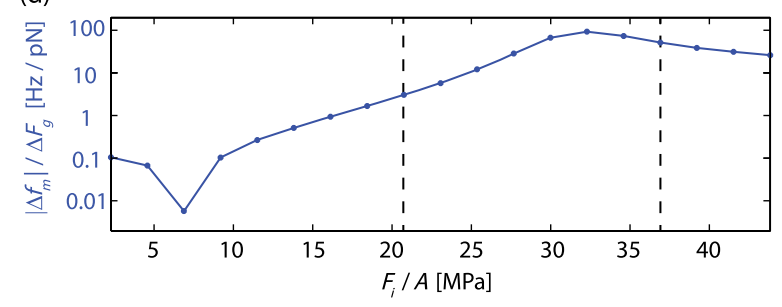

FIG. 12. (a) Longitudinal cross section of nanobeam used in ANSYS finite element simulations, showing the "arched" clampingpoint geometry. (b) Finite element simulations of resonance frequency and nanobeam deflection as a function of axial compressive stress. Dashed lines indicate value of $F_{i}$ where simulated $\omega_{m}$ approximately matches the experimentally observed value. (c) Simulated change in resonance frequency and nanobeam deflection for $P_{\mathrm{abs}}=1 \mu \mathrm{W}$ of absorbed optical power. (d) Simulated change in resonance frequency from a $F_{g}=1 \mathrm{pN}$ transverse load applied across the coupling region of the nanobeam (length $\left.L_{c}\right)$. Nanobeam dimensions are $L \times w \times d=80 \times 0.48 \times 0.25 \mu \mathrm{m}^{3}$, as in the self-oscillation measurements.

of the diamond substrate is fixed at constant temperature, and only conductive heat loss is considered. The corresponding temperature distribution is used to predict $R$ and $\kappa_{t}$, as summarized in Table I. The photothermal results are shown in Fig. 12(c), which plots the changes $\Delta \bar{x}$ and $\Delta \omega_{m}$ in deflection and frequency, respectively, for $P_{\text {abs }}=1 \mu \mathrm{W}$. These results clearly illustrate the sensitivity of photothermal effects on compressive stress and deflection and indicate that $\mathcal{F}$ varies by over 2 orders of magnitude depending on the compressive axial loading of the nanobeam.

Simulations of $\Delta \omega_{m}$ when a vertical transverse load mimicking the optical gradient force is distributed along the coupling region of the nanobeam (length $L_{c}$ ) are also performed and are shown in Fig. 12(d). These results show that $d \omega_{m} / d F_{g}$ varies by nearly 3 orders of magnitude depending on the compressive axial load. 
TABLE I. Parameters input to model for $\gamma_{m}^{\prime}(h)$ and $\omega_{m}^{\prime}(h)$. Values in brackets are predictions from simulations and are included for comparison with values determined from experimental fits.

\begin{tabular}{|c|c|c|c|}
\hline Parameter & $\begin{array}{l}\text { Input } \\
\text { value }\end{array}$ & Units & Source \\
\hline$\kappa_{t}$ & 1.4 & $\mu \mathrm{s}^{-1}$ & $\begin{array}{l}\text { Finite element } \\
\text { method (FEM) } \\
\text { simulation }\end{array}$ \\
\hline$R$ & 0.11 & $\mathrm{~K} / \mu \mathrm{W} \mu \mathrm{s}$ & FEM simulation \\
\hline$\omega_{m}^{i} / 2 \pi$ & 680 & $\mathrm{kHz}$ & FEM simulation \\
\hline$m$ & 10 & pg & FEM simulation \\
\hline $\bar{x}$ & -122 & $\mathrm{~nm}$ & FEM simulation \\
\hline$\alpha / 2 \pi$ & $-16(-28)$ & $\mathrm{Hz} / \mathrm{nm}^{2}$ & Fit (FEM) \\
\hline$C_{t}$ & 1.0 & $\mathrm{kHz} / \mu \mathrm{W}$ & FEM simulation \\
\hline$\zeta$ & 0.12 & $\mathrm{~cm}^{-1}$ & Fit \\
\hline $\mathcal{F} / k$ & -0.36 & $\mathrm{~nm} / \mu \mathrm{W}$ & FEM simulation \\
\hline $\mathcal{F}$ & -26 & $\mathrm{pN} / \mu \mathrm{W}$ & FEM simulation \\
\hline$F_{g}$ & Figure 11 & & $\begin{array}{l}\text { Optical mode } \\
\text { solver }\end{array}$ \\
\hline$d \omega_{m} / d F_{g}$ & $-7163(-377)$ & $\mathrm{rad} / \mathrm{s} \mathrm{pN}^{-1}$ & Fit (FEM) \\
\hline$P_{i}$ & 300 & $\mu \mathrm{W}$ & $\begin{array}{c}\text { Experimental } \\
\text { parameter }\end{array}$ \\
\hline$Q_{m}$ & 25000 & & $\begin{array}{l}\text { Experimental } \\
\text { parameter }\end{array}$ \\
\hline
\end{tabular}

\section{Dynamics: Small-amplitude oscillations}

For small mechanical oscillation amplitude, the power in the nanobeam waveguide can be approximated by $P_{n}=P_{n}\left(h_{o}\right)-\left.P_{i}(d T / d x)\right|_{h_{o}}[x(t)-\bar{x}]$. Inserting this into Eq. (G2) and retaining only terms that are linear in $x$ allows the Laplace transform of Eqs. (G1) and (G2) to be combined into a single linear equation:

$$
\begin{aligned}
- & \omega^{2} x(\omega)-i \frac{\omega_{m} \omega}{Q_{m}} x(\omega)+\omega_{m}^{2} x(\omega) \\
= & \frac{F_{s}(\omega)}{m}+\left(\frac{d F_{g}}{d x}+\frac{d F_{\mathrm{pt}}}{d x} \frac{i \omega \tau+1}{\omega^{2} \tau^{2}+1}\right) \frac{x(\omega)}{m},
\end{aligned}
$$

where constant force terms resulting in changes to static nanobeam deflection $\bar{x}$ have been left out for clarity but are included implicitly in $\omega_{m}(\bar{x})$. Rearranging terms reveals the optomechanical renormalization of the nanobeam dynamics,

$$
-\omega^{2} x(\omega)-i \gamma_{m}^{\prime} \omega x(\omega)+\omega_{m}^{\prime 2} x(\omega)=\frac{F_{s}(\omega)}{m},
$$

with

$$
\begin{aligned}
& \frac{\omega_{m}^{\prime 2}}{\omega_{m}^{2}}=1-\frac{1}{1+\omega^{2} \tau^{2}} \frac{d F_{\mathrm{pt}}\left(h_{o}\right)}{d x} \frac{1}{k}-\frac{d F_{g}\left(h_{o}\right)}{d x} \frac{1}{k}, \\
& \frac{\gamma_{m}^{\prime}}{\gamma_{m}}=1+Q_{m} \frac{\omega_{m} \tau}{1+\omega^{2} \tau^{2}} \frac{d F_{\mathrm{pt}}\left(h_{o}\right)}{d x} \frac{1}{k},
\end{aligned}
$$

where $d F_{\mathrm{pt}} / d x=-\mathcal{F} \zeta L_{i} P_{i} d T / d x$. Static effects modify the unperturbed resonance frequency $\omega_{m}^{o}$ according to

$$
\omega_{m}=\omega_{m}^{o}+C_{t} \zeta L_{i} P_{n}(h)+\frac{d \omega_{m}}{d F_{g}} F_{g}(h) .
$$

Equations (G10)-(G12) illustrate that combinations of mechanical softening or hardening and amplification or damping are possible. Higher-order thermoelastic effects [70] are predicted to be small and are not included in this analysis.

\section{Nonlinear dynamics: Large-amplitude oscillations}

When $\gamma_{m}^{\prime}$ approaches zero, the amplitude of mechanical oscillations about the static (i.e., buckled) position grows and nonlinear contributions to the system dynamics become significant. These nonlinear modifications originate from mechanisms intrinsic to the nanomechanical device geometry, or to the optomechanical response of the system, and are characterized here by nonzero $\alpha_{2,3}$ and $d^{n} T / d x^{n} \quad(n>1)$, respectively. Two nonlinear features observed in Fig. 5 are a softening in $\omega_{m}$ at the onset of self-oscillation and frequency harmonics in the selfoscillation region. While the latter effect is significantly affected by the nonlinear response of the optomechanical system, the analysis of Zaitsev et al. [70] shows that the optomechanical nonlinearity plays a negligible role in softening $\omega_{m}$ for the optomechanical system studied here. Rather, this softening is dominantly due to geometric nonlinearities of the deflected nanobeam.

Nonlinear coefficients $\alpha_{2}$ and $\alpha_{3}$ in Eq. (G1) can be derived from the Euler-Bernoulli equation for a nanobeam with static deflection $\bar{x}$, as in Refs. [83,84]. Using an approximate ansatz for the static nanobeam shape, expressions for $\alpha_{2}$ and $\alpha_{3}$ can be derived [71]. For oscillations about $\bar{x}$, if we group the nanobeam deformations into time-dependent and time-independent parts according to

$$
\phi(l, t)=(x(t)+\bar{x}) \frac{1}{2}(1-\cos (2 \pi l / L)),
$$

where $l$ is the coordinate running the length of the undeflected nanobeam, the Euler-Bernoulli equation yields Eq. (G1), with nonlinear coefficients

$$
\alpha_{2}=\bar{x} \frac{E}{6 \rho}\left(\frac{2 \pi}{L}\right)^{4} \frac{3}{8}
$$




$$
\alpha_{3}=\frac{E}{18 \rho}\left(\frac{2 \pi}{L}\right)^{4} \frac{3}{8}
$$

where $\rho$ is the density of the nanobeam material. Note that while $\alpha_{2}$ vanishes in a straight nanobeam, it is nonzero in deflected nanobeams $(|\bar{x}|>0)$.

Assuming that the solution to Eq. (G1) may be written as a combination of harmonic functions, the method of successive approximations [83-85] shows that to first order the fundamental frequency of oscillation is

$$
\begin{aligned}
\omega & =\omega_{m}(\bar{x})+\frac{v^{2}}{\omega_{m}(\bar{x})}\left(\frac{3}{8} \alpha_{3}-\frac{5}{12 \omega_{m}^{2}(\bar{x})} \alpha_{2}^{2}\right) \\
& =\omega_{m}(\bar{x})+v^{2} \alpha,
\end{aligned}
$$

where $v$ is the amplitude of oscillation and $\alpha$ is the effective nonlinear frequency shift coefficient. The first term in brackets is the well-known Duffing frequency modification, whereas the second term results from nonlinearities induced by static deflection of the nanobeam.

\section{Parameter estimation and comparison with experiment}

To compare the model described above with experimentally measured $\gamma_{m}^{\prime}(h)$, as shown in Fig. 5(c), a combination of known simulated and fit parameters are input into Eq. (G11), as summarized in Table I and described below.

For a given coupler operating condition, the photothermal force and the resulting $\gamma^{\prime}(h)$ described by Eq. (G11) scales linearly with $\zeta \mathcal{F}$. Neither $\mathcal{F}$ or $\zeta$ are known a priori. However, using finite element simulations to determine $\mathcal{F}$, as well as other parameters such as $\kappa_{t}$ and $R$, allows $\zeta$ to become the sole fitting parameter when comparing experimental and predicted values of $\gamma^{\prime}(h)$.

To determine $\mathcal{F}$ from finite element simulations, it is necessary to determine whether the nanobeam is in a prebuckled or postbuckled state. Insight into the buckling configuration of the nanobeam is provided by the observed nonlinear softening at the onset of self-oscillations, which is directly proportional to $v^{2}$ [Fig. 5(d)]. From these data and Eq. (G17), the nonlinear coefficient $\alpha$ given in Table I can be measured. Together with Eqs. (G14) and (G15), the corresponding nanobeam deflection amplitude of $|\bar{x}|=$ $98 \mathrm{~nm}$ is inferred, in good agreement with $\bar{x}=-122 \mathrm{~nm}$ predicted from finite element simulations of the nanobeam in its postbuckled configuration $\left(F_{i} / A \sim 37 \mathrm{MPa}\right)$, as shown in Fig. 12(b). When the nanobeam is in this postbuckled configuration, finite element simulations shown in Fig. 12(c) indicate that $\mathcal{F}=-26 \mathrm{pN} / \mu \mathrm{W}$ $(\mathcal{F} / k=-0.36 \mathrm{~nm} / \mu \mathrm{W})$. Negative $\mathcal{F}$ and $\bar{x}$ indicate that the photothermal force and nanobeam deflection, respectively, are in the down direction.
Inputing $\mathcal{F}$ and other finite element simulated parameters summarized in Table I into Eq. (G11), good agreement between predicted and observed $\gamma_{m}^{\prime}(h)$ is found for $\zeta \sim 0.12 \mathrm{~cm}^{-1}$. The corresponding optical absorption rate can be described by quality factor $Q_{o}=2 \pi n_{g} / \zeta \lambda$ $\sim 6.6 \times 10^{5}$, where the group index $n_{g} \sim 2.0$ of the nanobeam is predicted from numerical simulation. This absorption rate is smaller than combined absorption and radiation loss rates in other single-crystal diamond nanophotonic structures [47].

Given the value of $\zeta$ obtained from fitting $\gamma^{\prime}(h), \omega_{m}^{\prime}(h)$ predicted from Eq. (G10) can be compared with measurements. This is shown in Fig. 13, in which the predicted $\omega_{m}^{\prime}(h)$ is generated with $d \omega_{m} / d F_{g}$ as a fitting parameter, and other parameters set as in the model for $\gamma^{\prime}(h)$ and listed in Table I. Static and dynamic thermal effects are found to be significantly smaller than the maximum experimentally observed shift to $\omega_{m}$. Rather, the monotonic decrease in $\omega_{m}^{\prime}$, which becomes significant for $h<200 \mathrm{~nm}$, follows an $h$ dependence consistent with static tuning by the attractive optical gradient force $F_{g}(h)$ pulling up on a down-buckled nanobeam. This is in contrast to the static thermal tuning described by $C_{t}$, which is proportional to the power coupled into the nanobeam, and is expected to decrease in magnitude with decreasing $h$ for $h<200 \mathrm{~nm}$.

There is good agreement between the model and experimental observation in Fig. 13; however, the fit value for $d \omega_{m} / d F_{g}$ is larger than expected from finite element simulations [Fig. 12(d)] and predictions of $F_{g}(h)$ (Fig. 11). As shown in Fig. 12(d), $d \omega_{m} / d F_{g}$ is found in simulations to be highly variable, spanning over 3 orders of magnitude, depending on compressive stress and resulting buckling configuration. It is possible that imperfect nanobeam and clamping-point shape result in an enhanced sensitivity $[84,86]$. For example, including the triangular "arched" clamping points to approximate the fabricated structure, as shown in Fig. 12(a), enhances $d \omega_{m} / d F_{g}$ by a factor of $\sim 2$ compared to the case of ideal clamping points. Other effects not included here include reflection of light from the end of the waveguide resulting in enhanced optical interactions, dynamic and static displacement of the fiber taper due to optical forces [87], low-frequency fiber vibrations and possible parametric driving for small $h$, breakdown of

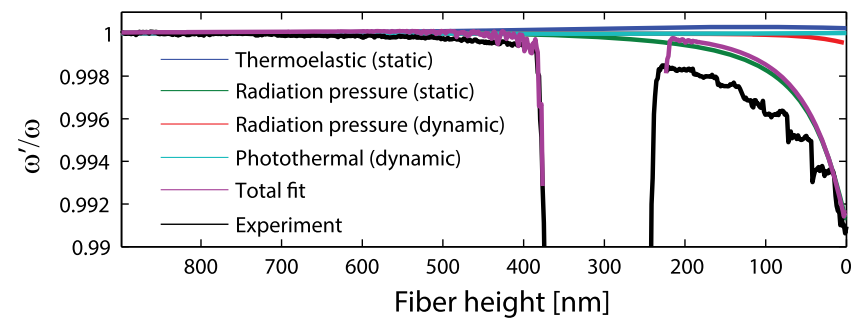

FIG. 13. Predicted $\omega_{m}^{\prime}(h)$ generated using the model in Eq. (G10) and the parameters in Table I. 
the coupler two-mode representation and the influence of higher-order modes for small $h$, and short-range effects such as the Casmir force [88].

In conclusion, the analysis we have presented here serves to illustrate the influence of compressive loading and buckling on the photothermal response of nanobeams and to show that significant photothermal forces are present at relatively low optical absorption levels. Note that imperfect nanobeams have significantly altered prebuckling and postbuckling behavior when subject to an compressive load [89], and taking into account these nonidealities is necessary to more accurately predict the nanobeam behavior.

[1] M. Li, W. H. P. Pernice, C. Xiong, T. Baehr-Jones, M. Hochberg, and H.X. Tang, Harnessing Optical Forces in Integrated Photonic Circuits, Nature (London) 456, 480 (2008).

[2] M. Eichenfield, J. Chan, R. M. Camacho, K. J. Vahala, and O. Painter, Optomechanical Crystals, Nature (London) 462, 78 (2009).

[3] E. Gavartin, R. Braive, I. Sagnes, O. Arcizet, A. Beveratos, T. J. Kippenberg, and I. Robert-Philip, Optomechanical Coupling in a Two-Dimensional Photonic Crystal Defect Cavity, Phys. Rev. Lett. 106, 203902 (2011).

[4] X. Sun, J. Zhang, M. Poot, C. W. Wong, and H. X. Tang, Femtogram Doubly Clamped Nanomechanical Resonators Embedded in a High-Q Two-Dimensional Photonic Crystal Nanocavity, Nano Lett. 12, 2299 (2012).

[5] O. Arcizet, P.-F. Cohadon, T. Briant, M. Pinard, A. Heidmann, J.-M. Mackowski, C. Michel, L. Pinard, O. Français, and L. Rousseau, High-Sensitivity Optical Monitoring of a Micromechanical Resonator with a Quantum-Limited Optomechanical Sensor, Phys. Rev. Lett. 97, 133601 (2006).

[6] A. Schliesser, O. Arcizet, R. Rivière, G. Anetsberger, and T. J. Kippenberg, Resolved-Sideband Cooling and Position Measurement of a Micromechanical Oscillator Close to the Heisenberg Uncertainty Limit, Nat. Phys. 5, 509 (2009).

[7] J. Chan, T. P. Mayer Alegre, A. H. Safavi-Naeini, J. T. Hill, A. Krause, S. Groblacher, M. Aspelmeyer, and O. J. Painter, Laser Cooling of a Nanomechanical Oscillator into Its Quantum Ground State, Nature (London) 478, 89 (2011).

[8] J. D. Cohen, S. M. Meenehan, and O. J. Painter, Optical Coupling to Nanoscale Optomechanical Cavities for Near Quantum-Limited Motion Transduction, Opt. Express 21, 11227 (2013).

[9] J. D. Teufel, T. Donner, M. A. Castellanos-Beltran, J. W. Harlow, and K. W. Lehnert, Nanomechanical Motion Measured with an Imprecision Below That at the Standard Quantum Limit, Nat. Nanotechnol. 4, 820 (2009).

[10] E. Verhagen, S. Deléglise, S. Weis, A. Schliesser, and T. J. Kippenberg, Quantum-Coherent Coupling of a Mechanical Oscillator to an Optical Cavity Mode, Nature (London) 482, 63 (2012).

[11] A. H. Safavi-Naeini, J. Chan, J. T. Hill, T. P. Mayer Alegre, A. Krause, and O. Painter, Observation of Quantum Motion of a Nanomechanical Resonator, Phys. Rev. Lett. 108, 033602 (2012).

[12] G. Anetsberger, O. Arcizet, Q.P. Unterreithmeier, R. Riviere, A. Schliesser, E. M. Weig, J. P. Kotthaus, and T. J. Kippenberg, Near-Field Cavity Optomechanics with Nanomechanical Oscillators, Nat. Phys. 5, 909 (2009).

[13] K. Srinivasan, H. Miao, M. T. Rakher, M. Davanço, and V. Aksyuk, Optomechanical Transduction of an Integrated Silicon Cantilever Probe Using a Microdisk Resonator, Nano Lett. 11, 791 (2011).

[14] A. G. Krause, M. Winger, T. D. Blasius, W. Lin, and O. Painter, A High-Resolution Microchip Optomechanical Accelerometer, Nat. Photonics 6, 768 (2012).

[15] M. Bagheri, M. Poot, M. Li, W. Pernice, and H. Tang, Dynamic Manipulation of Nanomechanical Resonators in the High-Amplitude Regime and Non-Volatile Mechanical Memory Operation, Nat. Nanotechnol. 6, 726 (2011).

[16] J. T. Hill, A. H. Safavi-Naeini, J. Chan, and O. Painter, Coherent Optical Wavelength Conversion via Cavity Optomechanics, Nat. Commun. 3, 1196 (2012).

[17] J. Bochmann, A. Vainsencher, D. D. Awschalom, and A. N. Cleland, Nanomechanical Coupling between Microwave and Optical Photons, Nat. Phys. 9, 712 (2013).

[18] A. Gruber, A. Dräbenstedt, C. Tietz, L. Fleury, J. Wratchtrup, and C. von Borczyskowski, Scanning Confocal Optical Microscopy and Magnetic Resonance on Single Defect Centers, Science 276, 2012 (1997).

[19] E. R. MacQuarrie, T. A. Gosavi, N. R. Jungwirth, S. A. Bhave, and G. D. Fuchs, Mechanical Spin Control of Nitrogen-Vacancy Centers in Diamond, Phys. Rev. Lett. 111, 227602 (2013).

[20] P. Ovartchaiyapong, K. W. Lee, B. A. Myers, and A. C. Bleszynski Jayich, Dynamic Strain-Mediated Coupling of a Single Diamond Spin to a Mechanical Resonator, Nat. Commun. 5, 4429 (2014).

[21] J. Teissier, A. Barfuss, P. Appel, E. Neu, and P. Maletinsky, Strain Coupling of a Nitrogen-Vacancy Center Spin to a Diamond Mechanical Oscillator, Phys. Rev. Lett. 113, 020503 (2014).

[22] E. R. MacQuarrie, T. A. Gosavi, A. M. Moehle, N. R. Jungwirth, S. A. Bhave, and G. D. Fuchs, Coherent Control of a Nitrogen-Vacancy Center Spin Ensemble with a Diamond Mechanical Resonator, Optica 2, 233 (2015).

[23] A. Barfuss, J. Teissier, E. Neu, A. Nunnenkamp, and P. Maletinsky, Strong Mechanical Driving of a Single Electron Spin, Nat. Phys. 11, 820 (2015).

[24] I. Yeo, P.-L. de Assis, A. Gloppe, E. Dupont-Ferrier, P. Verlot, N. S. Malik, E. Dupuy, J. Claudon, J.-M. Gerard, A. Auffves, G. Nogues, S. Seidelin, J. Ph. Poizat, O. Arcizet, and M. Richard, Strain-Mediated Coupling in a Quantum Dot-Mechanical Oscillator Hybrid System, Nat. Nanotechnol. 9, 106 (2013).

[25] M. Montinaro, G. Wüst, M. Munsch, Y. Fontana, E. RussoAverchi, M. Heiss, A. Morral, R. J. Warburton, and M. Poggio, Quantum Dot Opto-Mechanics in a Fully SelfAssembled Nanowire, Nano Lett. 14, 4454 (2014).

[26] O. Arcizet, V. Jacques, A. Siria, P. Poncharal, P. Vincent, and S. Seidelin, A Single Nitrogen-Vacancy Defect Coupled to a Nanomechanical Oscillator, Nat. Phys. 7, 879 (2011). 
[27] M. Wallquist, K. Hammerer, P. Rabl, M. Lukin, and P. Zoller, Hybrid Quantum Devices and Quantum Engineering, Phys. Scr. T137, 014001 (2009).

[28] L. Ding, C. Baker, P. Senellart, A. Lemaitre, S. Ducci, G. Leo, and I. Favero, High Frequency GaAs NanoOptomechanical Disk Resonator, Phys. Rev. Lett. 105, 263903 (2010).

[29] L. Sekaric, J. M. Parpia, H. G. Craighead, T. Feygelson, B. H. Houston, and J. E. Butler, Nanomechanical Resonant Structures in Nanocrystalline Diamond, Appl. Phys. Lett. 81, 4455 (2002).

[30] P. Ovartchaiyapong, L. M. A. Pascal, B. A. Myers, P. Lauria, and A. C. Bleszynski Jayich, High Quality Factor SingleCrystal Diamond Mechanical Eesonators, Appl. Phys. Lett. 101, 163505 (2012).

[31] Y. Tao, J. M. Boss, B. A. Moores, and C. L. Degen, SingleCrystal Diamond Nanomechanical Resonators with Quality Factors Exceeding One Million, Nat. Commun. 5, 3638 (2014).

[32] P. Rath, S. Khasminskaya, C. Nebel, C. Wild, and W. Pernice, Diamond-Integrated Optomechanical Circuits, Nat. Commun. 4, 1690 (2013).

[33] M. J. Burek, D. Ramos, P. Patel, I. W. Frank, and M. Lončar, Nanomechanical Resonant Structures in Single-Crystal Diamond, Appl. Phys. Lett. 103, 131904 (2013).

[34] T. Ramos, V. Sudhir, K. Stannigel, P. Zoller, and T. J. Kippenberg, Nonlinear Quantum Optomechanics via Individual Intrinsic Two-Level Defects, Phys. Rev. Lett. 110, 193602 (2013).

[35] P. Rabl, S. J. Kolkowitz, F. H. L. Koppens, J. G. E. Harris, P. Zoller, and M. D. Lukin, A Quantum Spin Transducer Based on Nanoelectromechanical Resonator Arrays, Nat. Phys. 6, 602 (2010).

[36] M. J. A. Schuetz, E. M. Kessler, G. Giedke, L. M. K. Vandersypen, M. D. Lukin, and J. I. Cirac, Universal Quantum Transducers Based on Surface Acoustic Waves, Phys. Rev. X 5, 031031 (2015).

[37] J. Cai, F. Jelezko, and M. B. Plenio, Hybrid Sensors Based on Colour Centres in Diamond and Piezoactive Layers, Nat. Commun. 5, 4065 (2014).

[38] S. D. Bennett, N. Y. Yao, J. Otterbach, P. Zoller, P. Rabl, and M. D. Lukin, Phonon-Induced Spin-Spin Interactions in Diamond Nanostructures: Application to Spin Squeezing, Phys. Rev. Lett. 110, 156402 (2013).

[39] I. Wilson-Rae, P. Zoller, and A. Imamoğlu, Laser Cooling of a Nanomechanical Resonator Mode to Its Quantum Ground State, Phys. Rev. Lett. 92, 075507 (2004).

[40] K. V. Kepesidis, S. D. Bennett, S. Portolan, M. D. Lukin, and P. Rabl, Phonon Cooling and Lasing with NitrogenVacancy Centers in Diamond, Phys. Rev. B 88, 064105 (2013).

[41] B. A. Fairchild, P. Olivero, S. Rubanov, A. D. Greentree, F. Waldermann, I. Walmsley, R. A. Taylor, J. M. Smith, S. Huntington, B. C. Gibson, D. N. Jamieson, and S. Prawer, Fabrication of Ultrathin Single-Crystal Diamond Membranes, Adv. Mater. 20, 4793 (2008).

[42] A. P. Magyar, J. C. Lee, A. M. Limarga, I. Aharonovich, F. Rol, D. R. Clarke, M. Huang, and E. L. Hu, Fabrication of Thin, Luminescent, Single-Crystal Diamond Membranes, Appl. Phys. Lett. 99, 081913 (2011).
[43] B. R. Patton, P. R. Dolan, F. Grazioso, M. B. Wincott, J. M. Smith, M. L. Markham, D. J. Twitchen, Y. Zhang, E. Gu, M. D. Dawson et al., Optical Properties of Single Crystal Diamond Microfilms Fabricated by Ion Implantation and Lift-Off Processing, Diam. Relat. Mater. 21, 16 (2012).

[44] A. Faraon, P. E. Barclay, C. Santori, K.-M. C. Fu, and R. G. Beausoleil, Resonant Enhancement of the Zero-Phonon Emission from a Color Center in a Diamond Cavity, Nat. Photonics 5, 301 (2011).

[45] I. Bayn, S. Mouradian, L. Li, J. A. Goldstein, T. Schröder, J. Zheng, E. H. Chen, O. Gaathon, M. Lu, A. Stein et al., Fabrication of Triangular Nanobeam Waveguide Networks in Bulk Diamond Using Single-Crystal Silicon Hard Masks, Appl. Phys. Lett. 105, 211101 (2014).

[46] J. Riedrich-Möller, L. Kipfstuhl, C. Hepp, E. Neu, C. Pauly, F. Mücklich, A. Baur, M. Wandt, S. Wolff, M. Fischer et al., One-and Two-Dimensional Photonic Crystal Microcavities in Single Crystal Diamond, Nat. Nanotechnol. 7, 69 (2012).

[47] M. J. Burek, Y. Chu, M. S. Z. Liddy, P. Patel, J. Rochman, S. Meesala, W. Hong, Q. Quan, M. D. Lukin, and M. Lončar, High Quality-Factor Optical Nanocavities in Bulk SingleCrystal Diamond, Nat. Commun. 5, 5718 (2014).

[48] K. A. Shaw, Z. Lisa Zhang, and N. C. MacDonald, SCREAM I: A Single Mask, Single-Crystal Silicon, Reactive Ion Etching Process for Microelectromechanical Structures, Sens. Actuators A 40, 63 (1994).

[49] B. Khanaliloo, M. Mitchell, A. C. Hryciw, and P. E. Barclay, High-Q/V Monolithic Diamond Microdisks Fabricated with Quasi-isotropic Etching, Nano Lett. 15, 5131 (2015).

[50] C. P. Michael, M. Borselli, T. J. Johnson, C. Chrystala, and O. Painter, An Optical Fiber-Taper Probe for Wafer-Scale Microphotonic Device Characterization, Opt. Express 15, 4745 (2007).

[51] A. Yariv and P. Yeh, Photonics: Optical Electronics in Modern Communications (Oxford, New York, 2006).

[52] P. E. Barclay, K. Srinivasan, M. Borselli, and O. Painter, Experimental Demonstration of Evanescent Coupling from Optical Fibre Tapers to Photonic Crystal Waveguides, Electron. Lett. 39, 842 (2003).

[53] M. Aspelmeyer, T. Kippenberg, and C. Marquardt, Cavity Optomechanics, Rev. Mod. Phys. 86, 1391 (2014).

[54] O. Basarir, S. Bramhavar, and K. L. Ekinci, Motion Transduction in Nanoelectromechanical Systems (NEMS) Arrays Using Near-Field Optomechanical Coupling, Nano Lett. 12, 534 (2012).

[55] M. Li, W. H. P. Pernice, and H. X. Tang, Broadband AllPhotonic Transduction of Nanocantilevers, Nat. Nanotechnol. 4, 377 (2009).

[56] D. Rugar, H. J. Mamin, and P.Guethner, Improved FiberOptic Interferometer for Atomic Force Microscopy, Appl. Phys. Lett. 55, 2588 (1989).

[57] D. T. Smith, J. R. Pratt, and L. P. Howard, A Fiber-Optic Interferometer with Subpicometer Resolution for $d c$ and Low-Frequency Displacement Measurement, Rev. Sci. Instrum. 80, 035105 (2009).

[58] B. W. Hoogenboom, P. L. T. Frederix, J. L. Yang, S. Martin, Y. Pellmont, M. Steinacher, S. Zäch, E. Langenbach, H.-J. Heimbeck, A. Engel et al., A Fabry-Perot Interferometer for Micrometer-Sized Cantilevers, Appl. Phys. Lett. 86, 074101 (2005). 
[59] M. Li, W. H. P. Pernice, and H. X. Tang, Reactive Cavity Optical Force on Microdisk-Coupled Nanomechanical Beam Waveguides, Phys. Rev. Lett. 103, 223901 (2009).

[60] S. Stapfner, L. Ost, D. Hunger, J. Reichel, I. Favero, and E. M. Weig, Cavity-Enhanced Optical Detection of Carbon Nanotube Brownian Motion, Appl. Phys. Lett. 102, 151910 (2013).

[61] M. Wu, A. C. Hryciw, C. Healey, D. P. Lake, H. Jayakumar, M. R. Freeman, J. P. Davis, and P. E. Barclay, Dissipative and Dispersive Optomechanics in a Nanocavity Torque Sensor, Phys. Rev. X 4, 021052 (2014).

[62] R. M. Cole, G. A. Brawley, V. P. Adiga, R. De Alba, J. M. Parpia, B. Ilic, H. G. Craighead, and W. P. Bowen, Evanescent-Field Optical Readout of Graphene Mechanical Motion at Room Temperature, Phys. Rev. Applied 3, 024004 (2015).

[63] A. Sawadsky, H. Kaufer, R. Moghadas Nia, S. P. Tarabrin, F. Ya Khalili, K. Hammerer, and R. Schnabel, Observation of Generalized Optomechanical Coupling and Cooling on Cavity Resonance, Phys. Rev. Lett. 114, 043601 (2015).

[64] F. Elste, A. A. Clerk, and S. M. Girvin, Quantum Noise Interference and Backaction Cooling in Cavity Nanomechanics, Phys. Rev. Lett. 102, 207209 (2009); 103, 149902 (E) (2009).

[65] I. Wilson-Rae, Intrinsic Dissipation in Nanomechanical Resonators due to Phonon Tunneling, Phys. Rev. B 77, 245418 (2008).

[66] Y.-I. Sohn, M. J. Burek, and M. Lončar, Dynamic Actuation of Single-Crystal Diamond Nanobeams, arXiv:1408.5822.

[67] C. H. Metzger and K. Karrai, Cavity Cooling of a Microlever, Nature (London) 432, 1002 (2004).

[68] I. Favero, C. Metzger, S. Camerer, D. König, H. Lorenz, J. P. Kotthaus, and K. Karrai, Optical Cooling of a Micromirror of Wavelength Size, Appl. Phys. Lett. 90, 104101 (2007).

[69] R. A. Barton, I. R. Storch, V. P. Adiga, R. Sakakibara, B. R. Cipriany, B. Ilic, S. P. Wang, P. Ong, P. L. McEuen, J. M. Parpia et al., Photothermal Self-Oscillation and Laser Cooling of Graphene Optomechanical Systems, Nano Lett. 12, 4681 (2012).

[70] S. Zaitsev, O. Gottlieb, and E. Buks, Nonlinear Dynamics of a Microelectromechanical Mirror in an Optical Resonance Cavity, Nonlinear Dyn. 69, 1589 (2012).

[71] I. Kozinsky, H. W. Ch Postma, I. Bargatin, and M. L. Roukes, Tuning Nonlinearity, Dynamic Range, and Frequency of Nanomechanical Resonators, Appl. Phys. Lett. 88, 253101 (2006).

[72] S. Gröblacher, J. T. Hill, A. H. Safavi-Naeini, J. Chan, and O. Painter, Highly Efficient Coupling from an Optical Fiber to a Nanoscale Silicon Optomechanical Cavity, Appl. Phys. Lett. 103, 181104 (2013).

[73] M. Poot, K. Y. Fong, M. Bagheri, W. H. P. Pernice, and H. X. Tang, Backaction Limits on Self-Sustained Optomechanical Oscillations, Phys. Rev. A 86, 053826 (2012).
[74] D. S. Hwang, T. Saito, and N. Fujimori, New Etching Process for Device Fabrication Using Diamond, Diam. Relat. Mater. 13, 2207 (2004).

[75] W. Bogaerts, P. De Heyn, T. Van Vaerenbergh, K. De Vos, S. K. Selvaraja, T. Claes, P. Dumon, P. Bienstman, D. Van Thourhout, and R. Baets, Silicon Microring Resonators, Laser Photonics Rev. 6, 47 (2012).

[76] B. D. Hauer, C. Doolin, K. S. D. Beach, and J. P. Davis, A General Procedure for Thermomechanical Calibration of Nano/Micro-Mechanical Resonators, Ann. Phys. (Amsterdam) 339, 181 (2013).

[77] M. Eichenfield, R. Camacho, J. Chan, K. J. Vahala, and O. Painter, A Picogram and Nanometer Scale Photonic Crystal Opto-Mechanical Cavity, Nature (London) 459, 550 (2009).

[78] J. Ma and M. L. Povinelli, Mechanical Kerr Nonlinearities due to Bipolar Optical Forces between Deformable Silicon Waveguides, Opt. Express 19, 10102 (2011).

[79] C. Metzger, I. Favero, A. Ortlieb, and K. Karrai, Optical Self Cooling of a Deformable Fabry-Perot Cavity in the Classical Limit, Phys. Rev. B 78, 035309 (2008).

[80] J. Restrepo, J. Gabelli, C. Ciuti, and I. Favero, Classical and Quantum Theory of Photothermal Cavity Cooling of a Mechanical Oscillator, C.R. Phys. 12, 860 (2011).

[81] M. T. A. Saif, On a Tunable Bistable MEMS-theory and Experiment, J. Microelectromech. Syst. 9, 157 (2000).

[82] D. Blocher, A. T. Zehnder, R. H. Rand, and S. Mukerji, Anchor Deformations Drive Limit Cycle Oscillations in Interferometrically Transduced MEMS Beams, Finite Elements in Analysis and Design 49, 52 (2012).

[83] A. M. Abou-Rayan, A. H. Nayfeh, D. T. Mook, and M. A. Nayfeh, Nonlinear Response of a Parametrically Excited Buckled Beam, Nonlinear Dyn. 4, 499 (1993).

[84] W. Lacarbonara, A. H. Nayfeh, and W. Kreider, Experimental Validation of Reduction Methods for Nonlinear Vibrations of Distributed-Parameter Aystems: Analysis of a Buckled Beam, Nonlinear Dyn. 17, 95 (1998).

[85] L. D. Landau and E. M. Lifshitz, Mechanics (ButterworthHeinemann, Washington, DC, 1976).

[86] H. M. Ouakad and M. I. Younis, The Dynamic Behavior of MEMS Arch Resonators Actuated Electrically, Int. J. Nonlinear Mech. 45, 704 (2010).

[87] M. Eichenfield, C. P. Michael, R. Perahia, and O. Painter, Actuation of Micro-optomechanical Systems via CavityEnhanced Optical Dipole Forces, Nat. Photonics 1, 416 (2007).

[88] W. H. P. Pernice, M. Li, D. Garcia-Sanchez, and H. X. Tang, Analysis of Short Range Forces in Opto-Mechanical Devices with a Nanogap, Opt. Express 18, 12615 (2010).

[89] W. Fang and J. A. Wickert, Post Buckling of Micromachined Beams, J. Micromech. Microeng. 4, 116 (1994). 